\title{
Gamma Oscillations in the Basolateral Amygdala: Localization, Microcircuitry, and Behavioral Correlates
}

\author{
Drew B. Headley, ${ }^{1}$ Pinelopi Kyriazi, ${ }^{1,2}{ }^{\oplus}$ Feng Feng, ${ }^{3}{ }^{\circledR}$ Satish S. Nair, ${ }^{3}$ and Denis Pare ${ }^{1}$ \\ ${ }^{1}$ Center for Molecular and Behavioral Neuroscience, Rutgers University, Newark, New Jersey 07102, ${ }^{2}$ Behavioral and Neural Sciences Graduate \\ Program, Rutgers University, Newark, New Jersey 07102, and ${ }^{3}$ Department of Electrical Engineering and Computer Science, University of Missouri, \\ Columbia, Missouri 65211
}

The lateral (LA) and basolateral (BL) nuclei of the amygdala regulate emotional behaviors. Despite their dissimilar extrinsic connectivity, they are often combined, perhaps because their cellular composition is similar to that of the cerebral cortex, including excitatory principal cells reciprocally connected with fast-spiking interneurons (FSIs). In the cortex, this microcircuitry produces gamma oscillations that support information processing and behavior. We tested whether this was similarly the case in the rat (males) LA and BL using extracellular recordings, biophysical modeling, and behavioral conditioning. During periods of environmental assessment, both nuclei exhibited gamma oscillations that stopped upon initiation of active behaviors. Yet, BL exhibited more robust spontaneous gamma oscillations than LA. The greater propensity of BL to generate gamma resulted from several microcircuit differences, especially the proportion of FSIs and their interconnections with principal cells. Furthermore, gamma in BL but not LA regulated the efficacy of excitatory synaptic transmission between connected neurons. Together, these results suggest fundamental differences in how LA and BL operate. Most likely, gamma in LA is externally driven, whereas in BL it can also arise spontaneously to support ruminative processing and the evaluation of complex situations.

Key words: basolateral amygdala emotion; fear; gamma oscillations; reward; synchrony

Significance Statement

The basolateral amygdala (BLA) participates in the production and regulation of emotional behaviors. It is thought to perform this using feedforward circuits that enhance stimuli that gain emotional significance and directs them to valence-appropriate downstream effectors. This perspective overlooks the fact that its microcircuitry is recurrent and potentially capable of generating oscillations in the gamma band $(50-80 \mathrm{~Hz})$, which synchronize spiking activity and modulate communication between neurons. This study found that BLA gamma supports both of these processes, is associated with periods of action selection and environmental assessment regardless of valence, and differs between BLA subnuclei in a manner consistent with their heretofore unknown microcircuit differences. Thus, it provides new mechanisms for BLA to support emotional behaviors.

\section{Introduction}

Accumulating evidence indicates that gamma oscillations support the contributions of basolateral amygdala nuclei (BLAs) to emotional behaviors. Emotionally arousing stimuli and contexts, whether conditioned or unconditioned, enhance gamma

Received Dec. 17, 2020; revised Apr. 4, 2021; accepted Apr. 6, 2021.

Author contributions: D.B.H., P.K., and D.P. designed research; D.B.H. and P.K. performed research; D.B.H., P.K., F.F., and S.S.N. analyzed data; F.F., D.B.H., and S.S.N. designed and performed the computational experiments; D.B.H. wrote the paper.

This work was supported by National Institute of Mental Health Grants R01-MH-112505 and R01-MH119854 to D.P. as well as Grant R01-MH-122023 to S.N.

The authors declare no competing financial interests.

Correspondence should be sent to Denis Paré at pare@rutgers.edu or Drew B. Headley at dbh60@newark. rutgers.edu.

https://doi.org/10.1523/JNEUROSCI.3159-20.2021

Copyright $\odot 2021$ the authors oscillations in the BLA (Pagano and Gault, 1964; Oya et al., 2002; Luo et al., 2007, 2009; Courtin et al., 2014; Amir et al., 2018). BLA gamma increases during the consolidation of aversive or appetitive memories (Kanta et al., 2019). Boosting or attenuating BLA gamma after training enhances or impairs subsequent memory strength (Huff et al., 2013; Kanta et al., 2019). Gamma oscillations entrain the activity of BLA principal neurons (PNs) more strongly than all other rhythms, causing them to fire in a more synchronized manner, while having little impact on their overall firing rates (FRs; Amir et al., 2018). Finally, the BLA contains PNs reciprocally connected with fast-spiking interneurons (FSIs) in a manner that is conducive to gamma generation through the pyramidal-interneuron network gamma (PING) mechanism (Whittington et al., 2000).

Although these results suggest that gamma supports the contribution of BLA to emotional behaviors, questions remain. 
Chief among them is whether the gamma oscillations observed in prior studies were related to emotional behaviors or the stimuli that triggered them. Indeed, conditioning paradigms typically constrain behavior to a single conditioned response (CR; e.g., freezing), giving the impression that conditioned stimuli (CSs) reflexively trigger CRs when in reality that is all the experimental conditions allow. Since conditioning increases the probability that a CS will elicit the obligatory CR and the two are temporally contiguous or overlapping, it remains unclear whether gamma oscillations support sensory processing, behavioral selection, or other cognitive processes.

Since the connections of the lateral (LA) and basolateral (BL) nuclei of the BLA differ, it is even possible that gamma supports sensory processing in LA and motor selection in BL. LA is the main recipient of sensory afferents from the thalamus (LeDoux, 2000; Sah et al., 2003), and BL has prominent reciprocal connections with the prefrontal cortex (McDonald, 1991) and hippocampus (Pitkänen et al., 2000) as well as direct projections to the striatum (Kita and Kitai, 1990). In addition, there is reason to expect that the mechanisms underlying the genesis of gamma differ between the two nuclei. Parvalbumin (PV)-expressing interneurons (a majority of which are FSIs; Woodruff and Sah, 2007), whose reciprocal interactions with PNs play a critical role in gamma genesis (Buzsáki and Wang, 2012), are less numerous in LA than BL (Smith et al., 1998; McDonald and Mascagni, 2001).

Therefore, the present study seeks to determine whether gamma oscillations are associated with the receipt of CSs or the emission of CRs and to compare the mechanisms underlying the genesis of gamma oscillations in LA and BL. To this end, we obtained simultaneous single-unit and local field potential (LFP) recordings in rats engaged in a recently introduced task [risk reward interaction (RRI) task; Kyriazi et al., 2018, 2020], where variations in the type and latency of CRs facilitate dissociation of neuronal events linked to CSs versus CRs. To gain further insights into the role of the microcircuitry underlying gamma generation, we also developed biophysical network models of LA and BL constrained by our data.

\section{Materials and Methods}

Experimental manipulations were conducted in compliance with the National Institutes of Health Guide for the Care and Use of Laboratory Animals and with the approval of the Institutional Animal Care and Use Committee of Rutgers University. Experiments were conducted in 11 Long-Evans rats (weight, 300-350 g; adult males; Charles River Laboratories; RRID:RGD_2308852) that were maintained on a $12 \mathrm{~h}$ light/dark cycle and housed individually. Before any procedure, rats were habituated to the animal facility for $\geq 7 \mathrm{~d}$ and then to handling 15 min daily for $\geq 3 \mathrm{~d}$.

There were two groups of rats. In the first group $(n=8)$, rats were first trained on the RRI task, then implanted with silicon probes and, $\geq 7 \mathrm{~d}$ after the surgery, retrained on the task. Recordings began when their performance reached presurgical levels (see below). To examine learning-dependent changes in gamma oscillations and perform current source density (CSD) analyses, three additional rats were first implanted with silicon probes and then trained on the task.

\section{Risk reward interaction task}

The apparatus consisted of a rectangular arena $(90 \times 30 \mathrm{~cm})$ with walls $(60 \mathrm{~cm})$ but no ceiling. Metal bars $(0.5 \mathrm{~mm})$ spaced $8 \mathrm{~mm}$ apart, served as the floor. The floor was divided into three sectors $(30 \times 30 \mathrm{~cm})$, each with light-emitting diodes (LEDs) underneath. At both ends of the arena, mounted on the walls, was a water port with an LED behind it. LED activation, for delivery of water through the water ports or of footshocks in each floor sector $(0.4 \mathrm{~mA}, 10 \mathrm{~s})$, was controlled by a programmable microcontroller (Arduino, SparkFun).

Activation of an LED for $20 \mathrm{~s}$ behind the active water port signaled availability of a water reward [ $60 \mu \mathrm{l}$; CS with reward (CS-R)]. The reward was delivered $10 \mathrm{~s}$ after the onset of the CS-R via a dipper that withdrew at CS-R offset. Likewise, activation of an LED (20 s) located below one of the floor sectors (CS-S) signaled that a mild footshock would be delivered through the floor of the corresponding sector $10 \mathrm{~s}$ after the onset of the CS-S. The CS-S and shock ended simultaneously. If rats were in the shock sector at CS-S onset, they could avoid the shock by moving to a different sector within $10 \mathrm{~s}$ of CS-S onset. If they were not in the shock sector at CS-S onset, they could passively avoid the shock. A video camera located above the apparatus recorded behavior at 29.97 frames/s. During task performance, white noise masked ambient sounds.

Before training in the RRI task, rats were placed on a water restriction protocol while ensuring that they remained at $\geq 85 \%$ of their initial body weight. Water restriction consisted of 6 consecutive days of restriction followed by $1 \mathrm{~d}$ of continuous access to water. Experiments were conducted during the light cycle.

On the day before the start of training, rats were habituated to the arena for $1 \mathrm{~h}$. During this habituation session, the white noise source was turned on but no CS, rewards, or shocks were administered. During the first $2 \mathrm{~d}$ of training, rats received two 15 min training sessions (6 CS$\mathrm{R}$ trials, 9 CS-S trials) separated by $4-6 \mathrm{~h}$. Once animals learned to avoid the shock, sessions were extended to $1 \mathrm{~h}$ (26 reward trials; 39 shock trials). Training continued on through days $3-7$, at which point rats generally reached $\geq 80 \%$ correct performance on all trial types combined.

\section{Surgical procedures}

Rats were implanted with silicon probes aimed at the BLA before $(n=3)$ or after $(n=8)$ training on the RRI task. Before and during this procedure, rats were anesthetized with isoflurane. They were administered atropine sulfate $(0.05 \mathrm{mg} / \mathrm{kg}$, i.m.) to aid breathing. They were placed in a stereotaxic apparatus with nonpuncture ear bars. The scalp was injected with a local anesthetic (bupivacaine, s.c.), and, 15 min later, an incision was made to expose the skull. A craniotomy was performed over the amygdala. One of two types of silicon probes (described below) was attached to a microdrive and aimed dorsal to the BLA (coordinates in millimeters relative to bregma: anteroposterior, -2.2 to -3.6 ; mediolateral, 5.2; dorsoventral, 6.0). Rats were allowed 1-2 weeks to recover from surgery, at which point recordings began. The probe was gradually lowered between recording sessions.

For animals implanted after reaching behavioral asymptote, the three types of silicon probes used were Buzsaki32L ( $n=3$ rats), Buzsaki64L ( $n=1$ rat), and a custom-designed probe ( $n=4$ rats). The Buzsaki32L and $64 \mathrm{~L}$ probes, respectively, consisted of four and eight shanks spaced $200 \mu \mathrm{m}$ apart. Recording sites were arranged in a tight "V"-shaped pattern at the tip. The custom-designed probe had four shanks with 16 channels on each shank arranged in a tetrode formation. Each tetrode was $333 \mu \mathrm{m}$ from the neighboring tetrode, and the intershank distance was $250 \mu \mathrm{m}$. For the animals implanted before training, we used silicon probes with an $8 \times 8$ grid of recording sites, with eight shanks and eight recording sites per shank. Shanks were spaced $200 \mu \mathrm{m}$ apart, and recording sites were distributed linearly along the shank also with $200 \mu \mathrm{m}$ spacing. All probes were manufactured by NeuroNexus.

\section{Behavioral analyses}

To track the position and head direction of the rat during the task, red and green LEDs were fixed to the rostral and caudal ends of the headcaps, respectively. The midpoint between the red and green LEDs indicated the head position whereas the angle between the midpoint and the red LEDs relative to the apparatus indicated the head direction. Behavior was analyzed using a custom-made graphical user interface in MATLAB (MathWorks). Behavior onset and end times were defined by indicating the first and last video frame when rats initiated and ended a behavior. For instance, the initiation of active avoidance corresponded to the first video frame when rats began to escape from the lit sector. The end of active avoidance was defined as the first video frame when rats concluded the avoidance behavior. We considered five behaviors, namely 
active avoidance (AA), passive avoidance (PA), freezing, reward approach, and reward anticipation.

\section{LFP processing, unit recordings, and clustering}

Recordings were obtained using 64-channel digitizing headstages (Intan Technologies) that were sampled at $30 \mathrm{kHz}$ and were stored on a hard drive. A high-pass filter was applied, followed by a median filter (window size, $1.1 \mathrm{~ms}$ ). Spikes were extracted with a threshold. Using KlustaKwik (http://klustakwik.sourceforge.net/), principal component analysis was applied on the action potentials, and the first three components were clustered. Then, using Klusters (Hazan et al., 2006), spike clusters were perfected manually. To reliably separate clusters, we computed autocorrelograms and cross-correlograms. Auto-correlograms were required to exhibit a refractory period $\geq 2 \mathrm{~ms}$. A refractory period in the cross-correlograms indicated that the same unit was shared between clusters. Units with unstable spike shapes were excluded.

To determine the duration of action potentials, we selected the channel with the highest peak-to-trough amplitude and measured the interval between peak and trough (Barthó et al., 2004). BLA cells were classified as presumed PNs or FSIs using the following two criteria: baseline firing rates and spike duration. BLA cells firing at $<6 \mathrm{~Hz}$ and spike duration $>0.55 \mathrm{~ms}$ were classified as PNs, cells firing $\geq 6 \mathrm{~Hz}$ and with spike durations $\leq 0.55 \mathrm{~ms}$ were classified as FSIs. Cells that did not meet the criteria for PNs or FSIs were not considered further. Striatal (STR) units were classified into FSIs or medium spiny neurons based on spike duration (trough-to-peak interval) and the distribution of interspike intervals. According to Berke (2008), neurons with $<2 \%$ of interspike intervals $>1 \mathrm{~s}$ were classified as FSIs. The trough-to-peak duration used for STR units was the same as for BLA cells $(0.55 \mathrm{~ms})$. Cells that met only one of the two criteria were not considered further. The interspike interval distribution indicated that very few spikes were separated by less than the refractory period (Hill et al., 2011). That is, 2.5, 50, and $97.5 \%$ of interspike intervals were $<4,13$, and $92 \mathrm{~ms}$, respectively. The signalto-noise distributions (Joshua et al., 2007) at 2.5, 50, and 97.5\% were $1.05,4.88$, and 13.44 , respectively.

\section{Extracellular recording analyses}

Local field potential processing. For LFPs, the raw extracellular signal from each cluster of recording sites (Buzsaki32L or 64L, eight in a V configuration, four in a tetrode configuration for the custom probe, or single sites on the $8 \times 8$ probe) was extracted by taking the median LFP signals. This signal was low-pass filtered (acausal, Butterworth, $300 \mathrm{~Hz}$ cutoff) and then downsampled to $1000 \mathrm{~Hz}$. To obtain CSD estimates from subjects with the $8 \times 8$ silicon probes, we used the inverse CSD (Łęski et al., 2011). In contrast to traditional CSD approaches that use the second spatial derivative of regularly spaced recording electrodes, the inverse CSD method instead creates a matrix that relates a set of recorded potentials to underlying current sources based on the positions of the electrodes. Inverting this equation yields the predicted current sources. In addition, a spline-based smoothing fit is applied to reduce spatial noise.

Spectral analyses. Spectral analyses were performed using wavelet decomposition. A family of complex Morlet wavelets $(\sigma=5)$ spanning $1-256 \mathrm{~Hz}$ in one-quarter octave steps were convolved with the LFP signals and corrected for inflation of amplitude at higher frequencies (Torrence and Compo, 1998). This yielded spectrograms that covered the entire recording session, sampled every millisecond. Converting the spectrogram to amplitudes was performed by taking the absolute value of the complex spectrogram, while the phase was derived from its angle. To calculate the deviation from $1 / f^{x}$, we log transformed the amplitude and performed a linear fit to that curve. Subtracting the fitted line revealed the percentage change of the spectrum from $1 / f^{x}$.

During trials, the interval between the onset of the CSs and behavioral responses varied. To average spectrograms across trials without blurring the different behavioral periods, we interpolated the spectrogram of each trial so that there were 20 time points within each behavioral epoch. This was done by dividing the start and stop periods of a behavioral epoch into 20 time bins, and then obtaining the average spectral power at each frequency for each time bin. The temporally interpolated trials of the same type (e.g., freezing followed by active avoidance) were averaged together for each electrode within a session.

Relationship between unit activity and field potentials. To determine the coordination between LFP gamma and spiking, each spike was associated with the nearest time point in the wavelet spectrogram. The gamma amplitude associated with each spike was taken from the mean spectral amplitude between 53 and $76 \mathrm{~Hz}$. The same was done for the phase, except that we calculated the circular mean. To restrict our analyses to periods with gamma bursts, we only analyzed spikes occurring when gamma power exceeded the 50th percentile. To estimate the entrainment of a neuron to gamma phase, 1000 spikes were extracted at random, the corresponding gamma phase of each spike was determined, and the pairwise phase consistency (PPC) index (Vinck et al., 2010) was calculated for this list. LFP data were obtained from an electrode at least $200 \mu \mathrm{m}$ away from the site where the single unit was recorded to avoid spike waveform contamination of phase estimates. When multiple sites were equally close, we performed the aforementioned calculations on each site and averaged the results.

Spike cross-correlation analyses. Single units recorded during the same session were subjected to cross-correlation analyses to identify putative monosynaptic connections. Spikes were sampled from the intertrial interval (ITI) period, to avoid spurious correlations induced by task-related activity. Cross-correlation functions were calculated over a $\pm 40 \mathrm{~ms}$ window with $0.33 \mathrm{~ms}$ bins. To account for biases introduced by the limited duration of each ITI period $(<30 \mathrm{~s})$ that inflate the spike coincidence counts near the center of the cross-correlation function, we trimmed spikes from the reference unit at the edges of ITI periods (Stark and Abeles, 2009).

Sharp peaks or troughs in the cross-correlation function near, but not at, $0 \mathrm{~ms}$ lag, may be indicative of monosynaptic connections. To test this, one wants to rule out that slow fluctuations in spike coincidences or the Poisson nature of spiking activity drive the observed peak. To estimate those contributions, we used a convolution strategy (Stark and Abeles, 2009), which was recently validated with ground truth measures of synaptic connections (English et al., 2017). For excitatory connections, we used a hollowed Gaussian convolution kernel with an SD of $3 \mathrm{~ms}$, while for inhibitory connections the SD was $10 \mathrm{~ms}$ (inhibition tends to be less sharp than excitation). At each time lag, the probability of the observed number of coincidences was estimated from the distribution expected from a Poisson process that had a rate equal to the convolved cross-correlation. Excitatory peaks were classified as putative monosynaptic excitatory connections if the $p$ value was $<0.01$ for at least two adjacent time bins $(0.66 \mathrm{~ms})$, and with a lag between 1 and $6 \mathrm{~ms}$. Inhibitory troughs were classified as putative monosynaptic inhibitory connections if the $p$ value was $<0.05$ for at least three adjacent time bins $(1 \mathrm{~ms})$ and had a lag between 1 and $10 \mathrm{~ms}$.

For pairs of units with putative excitatory monosynaptic connections, we estimated the effect of gamma phase on connection strength. Spikes from the postsynaptic unit were sorted based on whether they occurred during the peak or trough of the gamma cycle. Separate crosscorrelation functions (same procedure as above) were calculated for these two groups along with all the spikes from the presynaptic unit. Sampling spikes based on the phase of gamma can introduce artificial periodicities in the cross-correlation function, which can be eliminated by deconvolving, in the frequency domain, the cross-correlation function with the autocorrelation functions from both the presynaptic and postsynaptic units (Rosenberg et al., 1989; Headley and Weinberger, 2013). Once corrected, the peak in the cross-correlation function was measured as a proxy for effective connection strength.

\section{Histology}

At the conclusion of the experiments, rats were anesthetized with isoflurane. Small electrolytic lesions ( $10 \mu \mathrm{A}$ for $16 \mathrm{~s})$ were made on either the most ventral or dorsal electrodes, alternating between shanks, to ensure that the different shanks could be easily distinguished. Rats were then perfused/fixed transcardially, and their brains extracted and cut on a freezing microtome. Brain sections $(80 \mu \mathrm{m})$ were counterstained with thionin. Only cells determined histologically to have been recorded in the STR or BLA were considered. 
Table 1. Parameters related to synaptic connections

\begin{tabular}{|c|c|c|c|c|c|c|c|c|c|c|c|c|}
\hline \multirow[b]{2}{*}{ Parameters } & \multicolumn{4}{|l|}{ AMPA } & \multicolumn{4}{|l|}{ NMDA } & \multicolumn{4}{|l|}{ GABA } \\
\hline & $\begin{array}{l}\text { Reversal } \\
\text { potential } \\
\text { (mV) }\end{array}$ & $\begin{array}{l}\text { Rise/decay } \\
\text { time } \\
\text { constant } \\
\text { (ms) }\end{array}$ & $\begin{array}{l}\text { Conductance } \\
\text { (nS) }\end{array}$ & $\begin{array}{l}\text { Strength } \\
\text { (mean/ } \\
\text { variance) }\end{array}$ & $\begin{array}{l}\text { Reversal } \\
\text { potential } \\
(\mathrm{mV})\end{array}$ & $\begin{array}{l}\text { Rise/decay } \\
\text { time } \\
\text { constant } \\
\text { (ms) }\end{array}$ & $\begin{array}{l}\text { Conductance } \\
\text { (nS) }\end{array}$ & $\begin{array}{l}\text { Strength } \\
\text { (mean/ } \\
\text { variance) }\end{array}$ & $\begin{array}{l}\text { Reversal } \\
\text { potential } \\
(\mathrm{mV})\end{array}$ & $\begin{array}{l}\text { Rise/decay } \\
\text { time } \\
\text { constant } \\
\text { (ms) }\end{array}$ & $\begin{array}{l}\text { Conductance } \\
\text { (nS) }\end{array}$ & $\begin{array}{l}\text { Strength } \\
\text { (mean/ } \\
\text { variance) }\end{array}$ \\
\hline PN to PN & 0 & $0.3 / 6.9$ & 1 & $2 / 1$ & 0 & $3.7 / 125$ & 0.5 & $2 / 1$ & & & & \\
\hline PN to FSI & 0 & $0.1 / 2.4$ & 1 & $0.4 / 0.02$ & 0 & $3.7 / 125$ & 0.5 & $0.4 / 0.02$ & & & & \\
\hline $\begin{array}{l}\text { FSI to FSI (gap junction } \\
\text { coupling coefficient) }\end{array}$ & & & $\sim 0.01$ & & & & & & & & & \\
\hline
\end{tabular}

Kinetics of AMPA, NMDA, and GABA conductances used in the LA and BL computational models.

Table 2. Parameters related to extrinsic afferents

\begin{tabular}{|c|c|c|c|c|c|c|c|c|c|}
\hline \multirow[b]{2}{*}{$\begin{array}{l}\text { Neuron } \\
\text { type }\end{array}$} & \multirow[b]{2}{*}{$\begin{array}{l}\text { Mean } \\
\text { frequency }(\mathrm{Hz})\end{array}$} & \multicolumn{4}{|l|}{ AMPA } & \multicolumn{4}{|l|}{ NMDA } \\
\hline & & $\begin{array}{l}\text { Reversal } \\
\text { potential } \\
(\mathrm{mV})\end{array}$ & $\begin{array}{l}\text { Rise/decay } \\
\text { time } \\
\text { constant (ms) }\end{array}$ & $\begin{array}{l}\text { Conductance } \\
\text { (nS) }\end{array}$ & $\begin{array}{l}\text { Strength } \\
\text { (mean/ } \\
\text { variance) }\end{array}$ & $\begin{array}{l}\text { Reversal } \\
\text { potential } \\
(\mathrm{mV})\end{array}$ & $\begin{array}{l}\text { Rise/decay } \\
\text { time constant } \\
\text { (ms) }\end{array}$ & $\begin{array}{l}\text { Conductance } \\
\text { (nS) }\end{array}$ & $\begin{array}{l}\text { Strength } \\
\text { (mean/ } \\
\text { variance) }\end{array}$ \\
\hline for PNs & 2 & 0 & $0.5 / 7$ & 1 & $4 / 1$ & 0 & $5 / 125$ & 0.5 & $4 / 1$ \\
\hline for FSIs & 2 & 0 & $0.5 / 7$ & 1 & $0.5 / 0.01$ & 0 & $5 / 125$ & 0.5 & $0.5 / 0.01$ \\
\hline
\end{tabular}

Kinetics and reversal potentials used for the extrinsic synaptic inputs in the computational models of LA and BL.

Table 3:. Parameters related to point-conductance model

\begin{tabular}{|c|c|c|c|c|c|c|c|c|}
\hline \multirow[b]{3}{*}{ Neuron type } & \multicolumn{8}{|c|}{ Parameters } \\
\hline & \multicolumn{4}{|c|}{ Excitatory source } & \multicolumn{4}{|c|}{ Inhibitory source } \\
\hline & $g_{e 0}(n S)$ & $\partial_{e}(\mathrm{nS})$ & $\tau_{e}(\mathrm{~ms})$ & $E_{e}(\mathrm{mV})$ & $g_{i 0}(\mathrm{nS})$ & $\partial_{i}(\mathrm{nS})$ & $\tau_{i}(\mathrm{~ms})$ & $E_{i}(\mathrm{mV})$ \\
\hline for BL PN/FSI & $3.2 / 1.2$ & $3 / 0.1$ & $2.73 / 2.73$ & $0 / 0$ & $21 / 5.7$ & $8 / 2.6$ & $10.49 / 10.49$ & $-75 /-75$ \\
\hline
\end{tabular}

Parameters used for the point-conductance model.

Computational models of $L A$ and $B L$

The biophysical models for LA and BL each had 27,000 multicompartment neurons, and were adapted from our recent model (Feng et al., 2019). Here we provide only salient aspects of the models and the adaptations.

Single-cell models. Single-cell models were developed using the Hodgkin-Huxley formulation and a segregation technique (Alturki et al., 2016). The PN model has three compartments and 16 segments in total (soma with 1 segment: diameter, $15 \mu \mathrm{m}$; length, $15 \mu \mathrm{m}$; apical dendrite with 8 segments: diameter, $3 \mu \mathrm{m}$; length, $270 \mu \mathrm{m}$; proximal dendrites with 7 segments: diameter, $5 \mu \mathrm{m}$; length, $555 \mu \mathrm{m})$. The PNs had either high (type-A) or low (type-C) spike frequency adaptation in LA and BL nuclei. The FSI model had two compartments (soma with one segment: diameter, $15 \mu \mathrm{m}$; length, $15 \mu \mathrm{m}$; dendrite with one segment: diameter, $10 \mu \mathrm{m}$; length, $150 \mu \mathrm{m})$. Active and passive membrane properties for both types were matched to biological estimates (Faber et al., 2001; Sah et al., 2003).

Network size and cell type proportions. To reproduce the real neuron density in LA and BL nuclei, the size of the region was reduced from $1.4 \times 1.4 \times 1.4 \mathrm{~mm}^{3}$ in the study by Feng et al. $(2019)$ to $0.6 \times 0.6 \times 0.6$ $\mathrm{mm}^{3}$ for both models, keeping the cell numbers the same at 27,000. This resulted in a cell density of $1.25 \times 10^{5}$ neurons $/ \mathrm{mm}^{3}$, in the middle of the range from $2.5 \times 10^{4}$ to $2 \times 10^{5}$ neurons $/ \mathrm{mm}^{3}$ reported in rats (Tuunanen and Pitkänen, 2000; Salm et al., 2004; Pêgo et al., 2008; Rubinow and Juraska, 2009; Chareyron et al., 2011). Proportions of cell types were determined from experimental data. In LA, the composition was $89 \%$ PNs and $11 \%$ FSIs, and BL had $82 \%$ PNs and $18 \%$ FSIs; types A and $\mathrm{C}$ were in the ratio 64:26 in both models.

Intrinsic connections. The $\mathrm{PN} \rightarrow \mathrm{PN}, \mathrm{PN} \rightarrow \mathrm{FSI}$, and FSI $\rightarrow$ PN chemical synaptic connections in both models were revised from those in the study by Feng et al. (2019) and now follow distance-dependent connection probabilities based on our new in vivo experimental data. Chemical and electrical synaptic connections for FSI $\rightarrow$ FSI in both models were the same as that in the study by Feng et al. (2019). Axonal conduction delays on all connections were distance dependent. Synaptic parameters such as conductances, time constants, and weight distributions were kept identical for both models. See Table 1 for details.

Modeling of afferent drive and membrane fluctuations. Each model neuron received an extrinsic afferent in the form of a single independent Poisson input with mean frequency of $2 \mathrm{~Hz}$. To reproduce the membrane potential fluctuation seen in vivo, a point-conductance model was implemented in each single-cell model using an Ornstein-Uhlenbeck process (Destexhe et al., 2001). Point-conductance parameters for LA and BL nuclei were separately tuned to match experimental firing rates. For the hybrid models that explored the contribution of different microcircuit features to gamma genesis, point-conductance parameters were retuned to achieve correct firing rates. Parameters for afferent drive and the point-conductance model are listed in Tables 2 and 3.

Modeling the LFP. The extracellular potential (i.e., LFP) was modeled using the line source approximation method (Parasuram et al., 2016), with the electrode at the center of each model. To have a realistic neuron density with 27,000 modeled neurons, we sacrificed the model volumes of LA and BL nuclei by reducing them to $0.216 \mathrm{~mm}^{3}(0.6 \times 0.6 \times 0.6$ $\mathrm{mm}^{3}$ ), while these volumes in actual rats were reported to be $\sim 1.2 \mathrm{~mm}^{3}$ (Chareyron et al., 2011). Therefore, our models included fewer cells than in reality. However, for computing the LFP, such disparity cannot be neglected. To correct for this, we multiplied the LFP by a scaling factor derived using a method adapted from Linden et al. (2011). For the LA and BL models, we first systematically calculated different LFP amplitudes from different spherical volumes of neurons in the model, with radius spanning from 100 to $360 \mu \mathrm{m}$ in $20 \mu \mathrm{m}$ steps; $360 \mu \mathrm{m}$ was the equivalent spherical radius for the entire model structure. The scaling 
index was found by fitting a power law (Radius ${ }^{x}$, optimize $x$ ) between radius and LFP amplitude. Using this equation, the LFP scaling factor to compensate for the reduced size of our model can be derived using an approximation of the actual LA and BL nuclei volumes, which is a sphere with a $660 \mu \mathrm{m}$ radius. This resulted in the LFP scaling factors for LA and BL of 1.43 and 1.47, respectively. Note that the lower value of the scaling factor for LA was caused by less synchronization of neuronal activity.

\section{Model implementation}

We used the NEURON 7.6 simulator (Carnevale and Hines, 2005) for all our models. All simulations were run with a time step of $50 \mu$ s. Network models were run using the NeuroScience Gateway (NSG; www. nsgportal.org; Sivagnanam et al., 2013), and the Extreme Science and Engineering Discovery Environment (XSEDE; through allocation ID TG-IBN190005). Results were obtained by averaging five runs, each with varying random seeds for generating intrinsic connectivity, synaptic weights, and membrane fluctuation noise.

Quantifying model properties. To measure gamma strength in the model, a $1 / f^{x}$ curve was fit to the power spectrum based on values $\geq 20 \mathrm{~Hz}$, and the difference between the log-transformed original and fitted spectrums obtained. A difference of logs is equivalent to division, so the difference from $1 / f^{x}$ is expressed as the percentage deviation from $1 / f^{x}$. To account for the significant differences in power levels when comparing between $\mathrm{BL}$ and LA nuclei cases, we scaled the percentage deviation from $1 / f^{x}$ appropriately. The scale factor $S$ was calculated as $S=N / D$, with denominator $D=$ (spectral power in LA + spectral power in $\mathrm{BL}$ at that frequency), and numerator $N=$ (spectrum power at that frequency for LA or BL, depending on the case considered). The fullwidth at half-maximum measure was applied to the percentage deviation of the gamma spectrum curve.

\section{Statistical analyses}

Unless otherwise specified, data are reported as means with bootstrapestimated SEs. All $t$ tests were two sided, while $F$ tests were one sided. Depending on the type of data, different procedures were used to assess statistical significance, as indicated below. Note that for the results of permutation tests, we only report the probability of the observed data with respect to the permuted distribution. The reason for this is that degrees of freedom are not meaningful in permutation tests since the probability distribution is derived "empirically" (via a resampling scheme) and does not conform to any particular parametric probability distribution, which calculate the probability as a function of effect size and degrees of freedom (e.g., Student's $T, \chi^{2}$ ). Thus, permutation tests cannot be described in terms of degrees of freedom or effect size. The only relevant information is the permutation scheme and the probability that the permuted distribution generated the observed data point, both of which we report. For all other analyses, we also provide the sample sizes (e.g., number of recording sites, single units), degrees of freedom, and effect sizes.

Differences between spectrums and spectrograms. A linear model was run for each frequency, or time-frequency bin, with the dependent variable being the spectral amplitude (depending on the particular case: power, percentage deviation from $1 / f^{x}$, percentage deviation from power in the intertrial interval, or $z$ score), and the independent variable being a dummy-coded category whose effect we wished to assess (depending on the particular case: spectrum region of interest, early or late training sessions, or behavioral epoch). The results of these linear models provided a map of $t$ or $F$ statistics, that was then subjected to a mass univariate test. To do this, categories were permuted 1000 times, generating 1000 surrogate maps. Each surrogate map was thresholded at the $p<0.05$ level and the test statistics within each contiguously significant region were summed. The largest summed value was stored for each surrogate, generating a null distribution. The same thresholding was applied to the statistical map for the observed data, and summed test statistic for each region was then compared with the null distribution to get their $p$ value. The rationale for this approach is that statistical analysis of spectrograms is fraught for two reasons. First, there is a multiple-comparisons problem because the spectrogram has many time-frequency points, and standard corrections for this would result in unreasonable $p$ value thresholds. Second, and related to the first point, traditional multiplecomparisons approaches assume independence of the samples, which is not the case in spectrograms where adjacent time-frequency bins are highly correlated. Thus, we opted for a univariate mass statistic that is commonly used in neuroimaging studies and addresses both of these concerns. Essentially, it identifies regions of $F$ statistics whose summed $F$ value exceeds what would be expected if behavior had no effect (as established via permutation; see above).

Differences in circular distributions. For circular data, we used an ANOVA for circular data (Harrison and Kanji, 1988). If significant effects were found, these were followed up with post hoc comparisons using the common median test (Fisher, 1995).

Region and condition effects on univariate data. Mixed-effects linear models were performed with the animal ID as a random effect to control for subject-dependent differences and adjust the degrees of freedom for nested dependencies between observations (Aarts et al., 2014). In R, the lme4 package was used to calculate the mixed-effects models. These were subjected to an ANOVA with the Kenward-Roger degrees of freedom correction (lmertest package). If significant effects were found, post hoc tests were conducted on the estimated marginal means using Bonferroni-corrected $t$ tests (emmeans package).

Differences in proportions. To examine differences in proportions between regions, we used the Fisher exact test.

Differences between biophysical models. The gamma strength measured from different biophysical models was fit to a general linear model with dummy-coded terms for each BL microcircuit factor (firing rate, proportion of FSIs, inhibitory connectivity). The contribution of these factors was evaluated with an ANOVA. Because of the low number of data points per condition $(n=5)$, we obtained $p$ values for the ANOVA $F$ statistics by permuting 1000 times the design matrix passed to the general linear model that was then subjected to an ANOVA. This provided a null distribution that the observed $F$ statistics could be compared with.

\section{Data availability}

The full dataset and custom MATLAB code have not been deposited in a public repository, because of their size, but are available from the corresponding author on request. The LA and BL models are available for download from ModelDB (https://senselab.med.yale.edu/modeldb/).

\section{Results}

\section{Comparison between $\mathrm{BL}$ and LA gamma}

Rats $(n=8)$ were trained on the RRI task and then implanted with a movable silicon probe targeting the amygdala. We recorded LFPs and unit activity during performance of the RRI task. See Kyriazi et al. $(2018,2020)$ for the firing rate correlates of $\mathrm{BL}$ and LA neurons in the RRI task. To examine differences in the baseline expression of gamma oscillations, we compared $\mathrm{BL}$ and LA LFPs (recording sites: $n=77$ and 105, respectively) during the ITIs. As a control, we included STR sites $(n=112)$ whose gamma is consistent with volume conduction or imposition by afferent synapses (van der Meer et al., 2010; Carmichael et al., 2017). BL exhibited prominent bursts of gamma oscillations in the mid-gamma range (Fig. $1 A$, red trace), while gamma activity was less conspicuous in LA and STR (Fig. 1A, blue and black traces, respectively). Consistent with the visual inspection of the amplitude spectrum, comparing the average spectrum of LFPs recorded at the three sites (Fig. $1 B$ ) revealed that gamma power in the $53-107 \mathrm{~Hz}$ range was significantly higher in BL than in LA or STR (univariate mass test, $p=0.002$ ).

Although the above indicates that gamma oscillations have a higher amplitude in BL than in LA or STR, it remains unclear whether they are locally generated; they could be volume conducted from structures neighboring BL. To determine whether the gamma oscillations seen in BL LFPs are in fact associated with local transmembrane currents, in a separate set of rats $(n=3)$ implanted with an $8 \times 8$ recording site grid we performed 

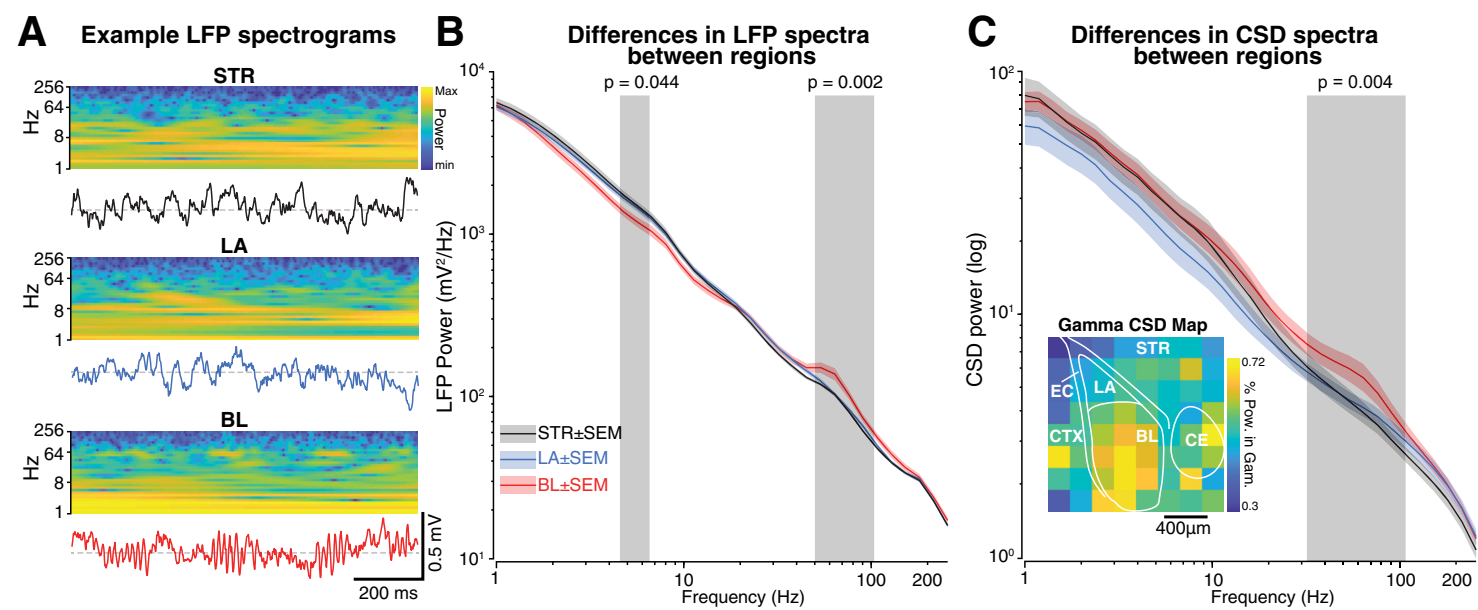

Figure 1. Gamma oscillations are more prominent in BL than in LA or STR. A, Representative examples of LFPs (traces) and corresponding spectrogram (colored plots) for the STR (top), LA (middle), and BL (bottom). Data obtained from eight rats during intertrial intervals (112 in STR; 105 in LA; and 77 in BL). B, Average \pm SEM power spectrum for BL (red; $n=77$ ), LA (blue; $n=105$ ), and STR (black, $n=112$ ). Gray-shaded regions mark significantly different consecutive bins. C, Average \pm SEM (SD power spectrum (three rats) for BL (red), LA (blue), and STR (black). Gray-shaded region marks significantly different consecutive bins in the gamma range. Data were obtained outside the task context, during epochs of quiet wakefulness.

CSD analysis. As illustrated in Figure $1 C$, gamma power in the CSD power spectrum, which reflects locally generated current sinks and sources, was significantly higher in BL than in adjacent regions (univariate mass test, $p=0.004$ ). Furthermore, these recordings were obtained in subjects outside the training context during a quiet waking state, suggesting that the differences seen between LA and BL are not specific to the state of arousal.

\section{Firing entrainment by gamma}

Given the CSD results, one would expect gamma oscillations to entrain unit activity more strongly in BL than in LA or STR. To test this, we recorded units (Fig. 2A1) in the three regions and compared their entrainment by gamma (STR PNs, 165; LA PNs, 264; BL PNs, 208; STR FSIs, 44; LA FSIs, 31; BL FSIs, 45). As in prior studies (Berke, 2008; Amir et al., 2018; Kyriazi et al., 2018), $\mathrm{BL}$ and LA neurons were classified as putative PNs or FSIs based on their firing rates and spike duration (Fig. 2A2,A3; see Materials and Methods). To prevent spurious phase locking arising from LFP contamination by spike waveforms (Zanos et al., 2011; Scheffer-Teixeira et al., 2013), we referenced spiking activity to LFP gamma recorded from the nearest adjacent recording site (typically, $200 \mu \mathrm{m}$ away from the unit being considered). The wavelet spectral phase and amplitude were taken in the LFP between 53 and $76 \mathrm{~Hz}$, where the gamma power spectrum peaked (Fig. 1B), and were used to calculate the coordination of spiking activity with gamma phase and amplitude.

In keeping with our analyses of gamma power (Fig. 1), PNs (Fig. 2B1, red) and FSIs (Fig. 2B1, blue) were more strongly entrained by gamma in BL than LA or STR. On average, there was a greater firing rate modulation by gamma in BL than LA or STR (Fig. 2B1). When stratifying gamma by its amplitude, the same trend was evident (Fig. 2B2). To quantify this, we calculated the entrainment of each unit to gamma using the PPC score (Vinck et al., 2010; Fig. 2C). A mixed-effects ANOVA comparing gamma entrainment as a function of recording site and cell type revealed a significant effect of recording site $\left(F_{(2,748.96)}=48.17\right.$, $\left.p<2 \times 10^{-16}\right)$ and an interaction between location and cell type $\left(F_{(2,745.16)}=5.41, p=0.0046\right)$. Post hoc tests using estimated marginal means confirmed that $\mathrm{PNs}$ were significantly more entrained in BL than LA $\left(t_{(751)}=6.28, p<0.0001\right)$ and STR $\left(t_{(751)}=6.65, p<0.0001\right)$. However, the entrainment of PNs in
LA and STR did not differ significantly $\left(t_{(747)}=0.86, p=1.0\right)$. A similar pattern of was observed for FSIs (BL vs LA: $t_{(746)}=5.35$, $p<0.0001$; BL vs STR: $t_{(746)}=7.3, p<0.0001$; LA vs STR: $t_{(746)}=$ $1.21, p=1.0$ ), with presumed interneurons being significantly more entrained in BL than in LA and STR.

Our analyses also revealed significant differences in preferred gamma firing phase (Fig. $2 D$ ). Whereas on average PNs in $\mathrm{BL}$ and LA preferentially fired just before the trough of gamma, FSIs tended to fire shortly after the trough of gamma. An ANOVA for circular data (Harrison and Kanji, 1988) comparing gamma firing phase as a function of recording site and cell type revealed a significant effect of recording site $\left(\chi^{2}(4)=49.15, p=\right.$ $\left.5.43 \times 10^{-10}\right)$ and cell type $\left(\chi^{2}(2)=11.21, p=0.004\right)$ as well as a significant interaction between these two variables $\left(\chi^{2}(2)=\right.$ $\left.14.60, p=6.76 \times 10^{-4}\right)$. Post hoc common median tests (Fisher, $1995)$ confirmed that BL and LA PNs fired significantly earlier in the gamma cycle than FSIs $(p(1)=4.95, p=0.026)$. This was in contrast with PNs and FSIs of the STR, whose preferred firing phase did not differ significantly $(p(1)=0.001, p=0.97)$.

Since neurons in BLA showed stronger entrainment to gamma than those in STR, and also exhibited phase locking consistent with local generation arising from reciprocal interactions between PNs and FSIs, all further analyses focused on the BLA.

\section{Influence of gamma on the efficacy of putative monosynaptic connections}

Prior in vivo whole-cell recordings have revealed that periodic fluctuations in inhibitory drive promote neuronal entrainment to gamma in cortex (Atallah and Scanziani, 2009; Salkoff et al., 2015). Since inhibition raises the threshold for excitatory inputs to evoke action potentials, gamma phase should modulate the sensitivity of neurons to excitatory inputs. Given the predominance of gamma in BL over LA, this effect should be strongest in BL. To test these predictions, we assessed how gamma modulates spike transmission between connected pairs of neurons. We calculated the cross-correlation function between all pairs of single units in either LA or BL, and identified putative excitatory monosynaptic connections (Stark and Abeles, 2009; English et al., 2017; Fig. 3A).

We focused our analyses on two types of excitatory connections, $\mathrm{PN} \rightarrow \mathrm{PN}$ and $\mathrm{PN} \rightarrow$ FSI. For connected pairs $(\mathrm{PN} \rightarrow \mathrm{PN}$ : 

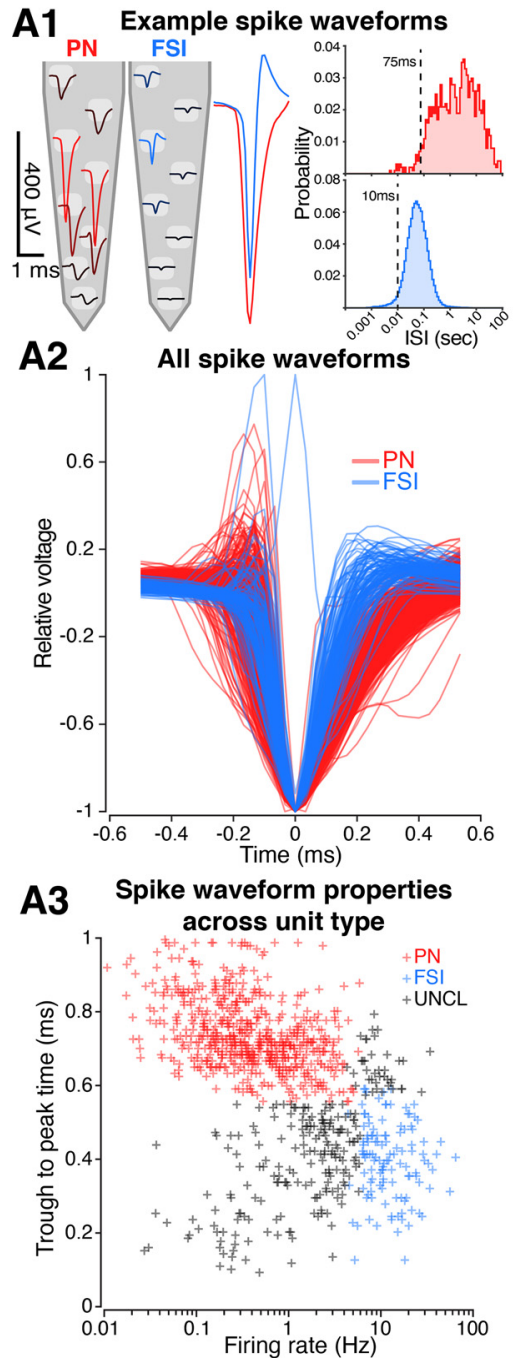

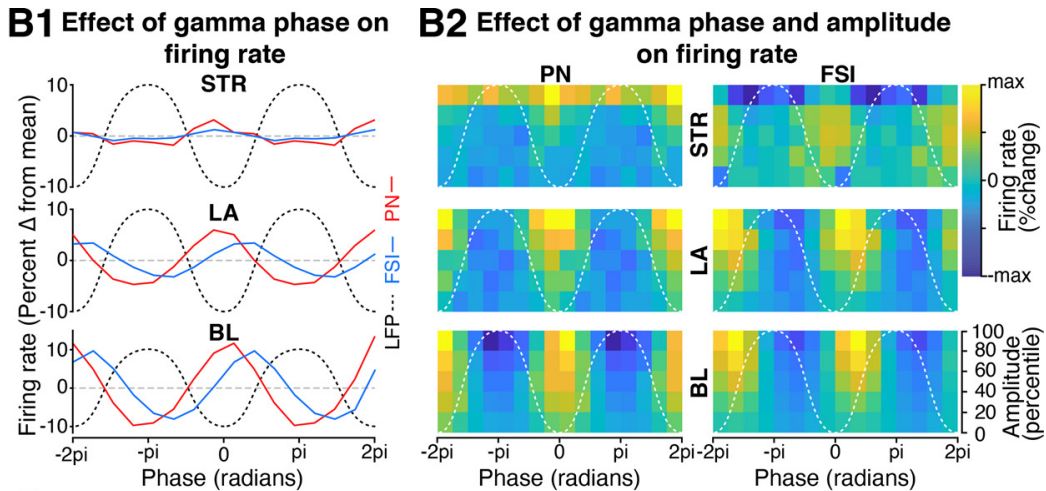

C

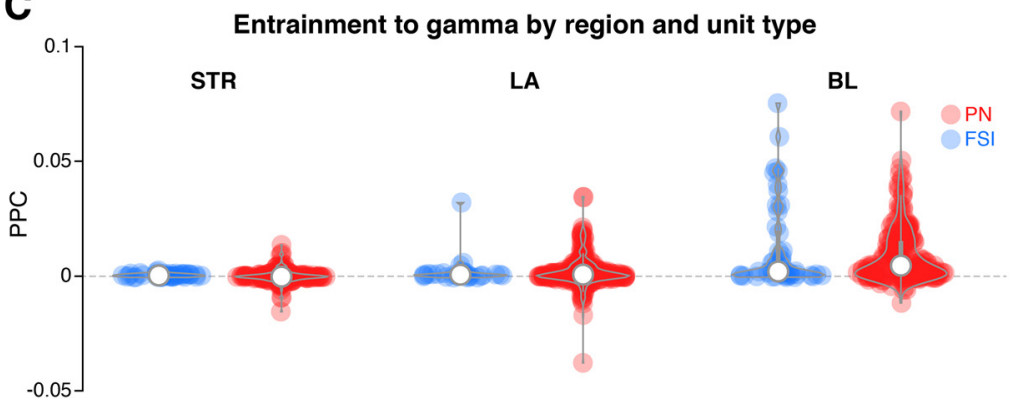

D

Preferred gamma phase by region and unit type

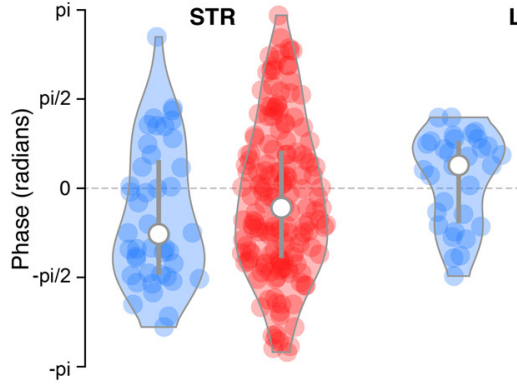

BL

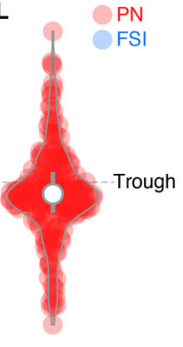

Figure 2. Gamma oscillations entrain BL neurons more strongly than LA or STR cells. A1-A3, Classification of presumed PNs and FSIs. A1, Left, Average spike shape of a particular PN or FSI as seen on different recording leads of a silicon probe. Red and blue are used for the principal cell and FSI, respectively. The degree of redness or blueness at each lead is proportional to spike amplitude. Middle, Overlaid spikes of the same PN and FSI. Right, Interspike interval (ISI) histograms for the two units shown. Dashed vertical line indicates the ISI below which at least 5\% of ISIs occurred. A2, Overlay of the average spike shape of all PNs (red) and FSIs (blue) included in this study. A3, Trough to peak time plotted as a function of firing rates for PNs (red), FSIs (blue), and unclassified neurons (UNCL; 72 BL, 94 LA, 60 STR cells). Same neurons as in A2. B1, Modulation of firing rate as a function of gamma phase for STR (top), LA (middle), and BL (bottom) PNs (red) and FSIs (blue). B2, Same as B1 but after stratifying the data by gamma amplitude (PNs, left; FSIs, right). C, Entrainment (PPC; see Materials and Methods) of STR (left), LA (middle), and BL (right) FSIs (blue) and PNs (red). The width of the violin plots is proportional to the concentration of the data at the different PPC values. White circles correspond to the median, and the gray lines originating from them indicate the PPC values comprising the middle $25-75 \%$ of the distribution. D, Preferred gamma firing phase of STR (left), LA (middle), and BL (right) FSIs (blue) and PNs (red). The width of the violin plots is proportional to the concentration of the data at the different phase values. See $\boldsymbol{C}$ for meaning of white circles and gray lines.

$\mathrm{BL}, n=21$; LA, $n=25 ; \mathrm{PN} \rightarrow$ FSI: BL, $n=97$; LA, $n=32$ ), we sorted presynaptic spikes by whether they occurred during the peak or trough period of gamma bursts (amplitude greater than median power) and used these to calculate two separate crosscorrelation functions, while correcting for phase-dependent changes in firing rate (see Materials and Methods). As predicted, many $\mathrm{PN} \rightarrow \mathrm{PN}$ and $\mathrm{PN} \rightarrow$ FSI pairs had higher cross-correlation peaks during the trough of gamma (Fig. $3 B$ ), when inhibitory drive should be lowest (Hasenstaub et al., 2005). A mixedeffects ANOVA determined whether cross-correlation strength was affected by gamma phase, connection type, and region. It revealed a significant interaction between gamma phase and region $\left(F_{(2,337.92)}=27.94, p=2.2 \times 10^{-7}\right.$; Fig. $\left.3 C\right)$. This interaction was clarified by post hoc tests, which found that the crosscorrelation for both pair types in BL were stronger during the trough of gamma $\left(\mathrm{PN} \rightarrow \mathrm{PN}: t_{(337.9)}=4.72, p=0.0001 ; \mathrm{PN} \rightarrow\right.$ FSI: $\left.t_{(337.9)}=6.12, p<0.0001\right)$, but this was not the case in LA $\left(\mathrm{PN} \rightarrow\right.$ PN: $t_{(337.9)}=-1.27, p=1.0 ; \mathrm{PN} \rightarrow$ FSI: $t_{(337.9)}=0.022$, $p=1.0$ ). Thus, $\mathrm{BL}$ gamma phase modulates excitatory synaptic transmission.

\section{Differences in intrinsic connectivity between $\mathrm{BL}$ and LA}

Overall, the results described so far indicate that gamma oscillations are more prominent in $\mathrm{BL}$ than $\mathrm{LA}$, entrain $\mathrm{BL}$ neurons more strongly, and cause a more pronounced modulation of their excitatory interactions. The origin of these differences is unclear, however. We examined whether LA and BL differed (1) in the amount of excitation present in their respective networks (as indexed by the firing rates of PNs), (2) in the connections between FSIs and PNs, and (3) in the incidence of FSIs. 


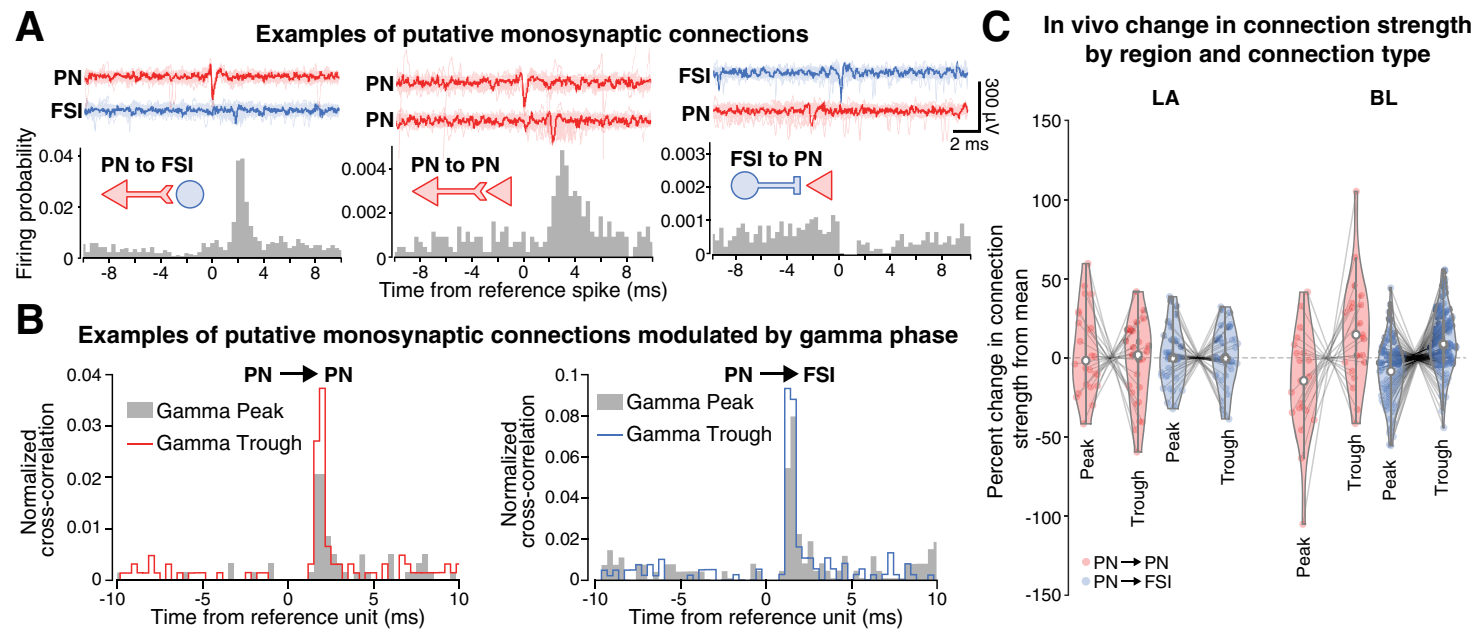

Figure 3. Modulation of putative monosynaptic connections by gamma phase. $A$, Examples of three types of connections identified by cross-correlation analyses. Top colored traces are unitfiltered extracellular spike traces, with a prototypical trace unshaded. Below the histogram, the probability of postsynaptic cell spiking relative to when the presynaptic cell spiked is shown. An excitatory connection from a PN $\rightarrow$ FSI (left) and a PN $\rightarrow$ PN (middle), and inhibitory connection from an FSI $\rightarrow$ PN (right). B, Examples of putative monosynaptic connections from PN $\rightarrow$ $\mathrm{PN}$ (left) and PN $\rightarrow$ FSI (right) that were modulated by gamma. C, Paired violin plot showing changes in cross-correlation strength as a function of region (LA, left; BL, right) and postsynaptic cell type (PN, red; FSI, blue). Each faded line corresponds to a single putative excitatory monosynaptic connection. White circles correspond to the median and gray lines to the 25th to 75th percentiles.

The incidence of FSIs was significantly higher in BL than LA (Fig. $4 A$; 17.8 vs $10.5 \%$; Fisher exact test, two-sided, $p=0.018$ ). As was previously reported (Paré and Gaudreau, 1996), PNs tended to fire at lower rates in LA than in BL, but this difference did not reach significance in our samples (Fig. $4 B ; 0.61$ vs $0.95 \mathrm{~Hz}$; mixed-effects model, $\left.F_{(1,283.08)}=0.77, p=0.38\right)$. Most importantly, the proportion of putative PN $\rightarrow$ FSI and FSI $\rightarrow$ PN (Fig. 4C1) monosynaptic connections was significantly higher in $\mathrm{BL}$ than in LA (Fisher exact test; PN $\rightarrow$ FSI, $p=$ $6.17 \times 10^{-5}$; FSI $\left.\rightarrow \mathrm{PN}, p=6.68 \times 10^{-9}\right)$. In contrast, no difference was found in the connections between PNs ( $\mathrm{PN} \rightarrow \mathrm{PN}$, $p=0.55$ ). Crucially, these connection probabilities were in agreement with estimates from paired-patching experiments on in vitro amygdala slices (Woodruff and Sah, 2007). Yet, in both nuclei, connection probabilities fell off with distance (Fig. 4C2C4), consistent with cortical networks that produce gamma (Oswald et al., 2009).

\section{Effect of microcircuit differences on gamma generation in biophysical models}

The differences we observed in microcircuitry between LA and BL may underlie their differing propensity to generate gamma. To evaluate which of these properties impacts gamma generation, we constructed biophysical network models of LA and BL that incorporated the differences in FSI incidence, PN firing rates, and connectivity described above. Simulations with each set of microcircuit parameters were run five times to obtain a representative sample of network behavior. Each network was composed of 27,000 multicompartmental biophysical model neurons with packing density matching stereological estimates (Chareyron et al., 2011). Membrane noise was titrated to match the firing rates of PNs and FSIs in vivo. Given the transmembrane current and location of each cellular compartment, we could emulate the LFP recorded at the center of the structure using the line source approximation method (Parasuram et al., 2016). To facilitate comparison between the model and in vivo power spectrums, we measured the deviation of each one from a fitted $1 / f^{x}$ curve (also termed "power law fit"), generating a residual spectrum. Indeed, when integrated over a long time period, LFP power shows a characteristic attenuation with frequency, which can be fitted with a $1 / f^{x}$ curve. During sensory, motor, or cognitive processing, the power spectrum deviates from the $1 / f^{x}$ curve as bumps or peaks become apparent in particular frequency bands. Subtracting the power law fit from the actual power spectrum thus allowed us to isolate the impact of various manipulations on mid-gamma power.

The mid-gamma band of the model spectrums lay within the $95 \%$ confidence intervals of the in vivo spectrums (LA, 42nd percentile; BL, 44th percentile; Fig. 4D). Importantly, the residual spectrum of the LA model did not show a bump in the midgamma band, while the BL model did. Thus, the peak power in each of these models fell near the middle of the in vivo distribution.

While these simulations suggest that microcircuit differences between LA and BL support their differing propensity to gamma generation, it is unclear which factor or factors are critical. To explore this, we systematically added BL microcircuit properties to the LA model one or two at a time and assessed the impact of these manipulations on gamma power, as measured in the resulting residual spectrum (Fig. $4 E, F$ ). Firing rates of PNs and FSIs were kept consistent across all hybrid models by making adjustments solely to the background noise levels, if needed. An ANOVA revealed that no single factor is responsible for the higher gamma levels seen in BL. Incorporating just the BL connectivity between PNs and FSIs caused a significant, but modest, increase in LA gamma (ANOVA, permutation $p=0.014$ ), while neither firing rate nor proportion of FSIs had a significant effect $(p=0.54$; FSI proportion, $p=0.052)$. However, their combination significantly increased gamma (permutation, $p=0.001$ ). The combination of BL connectivity and percentage of FSIs strengthened LA gamma further $(p=0.001)$, to the point where it was on par with that found in $\mathrm{BL}(p=0.18)$.

\section{Learning-related changes in oscillatory activity during intertrial intervals}

Having explored the physiology underlying the generation of gamma oscillations in the BLA, we sought to understand their 
A Proportion of B Distribution of PN cell types

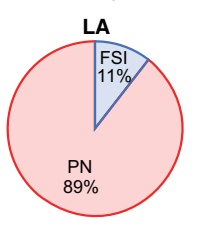

firing rates by region

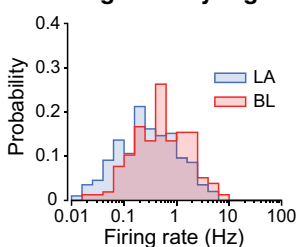

Distribution of FSI
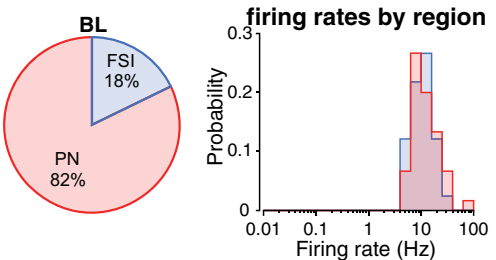
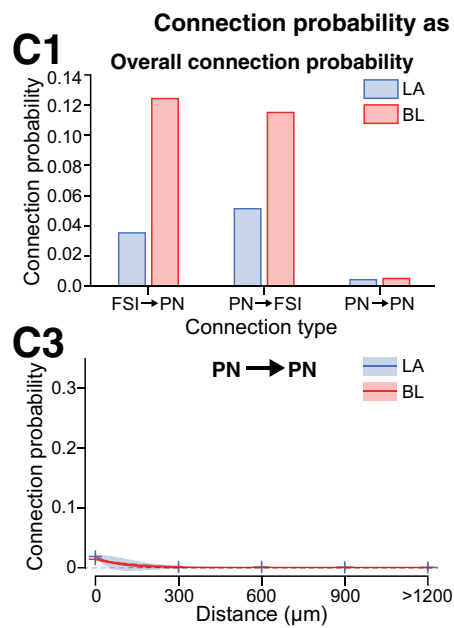
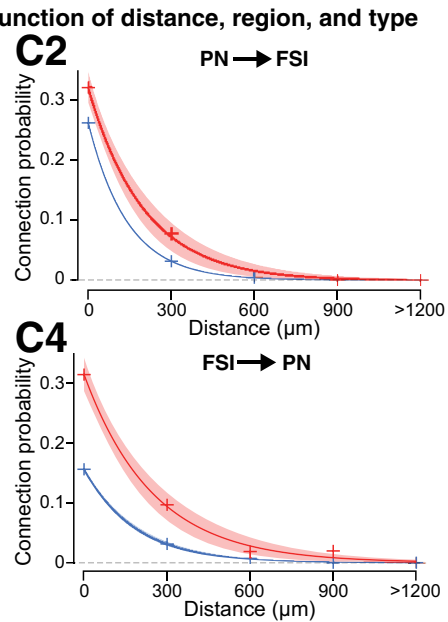
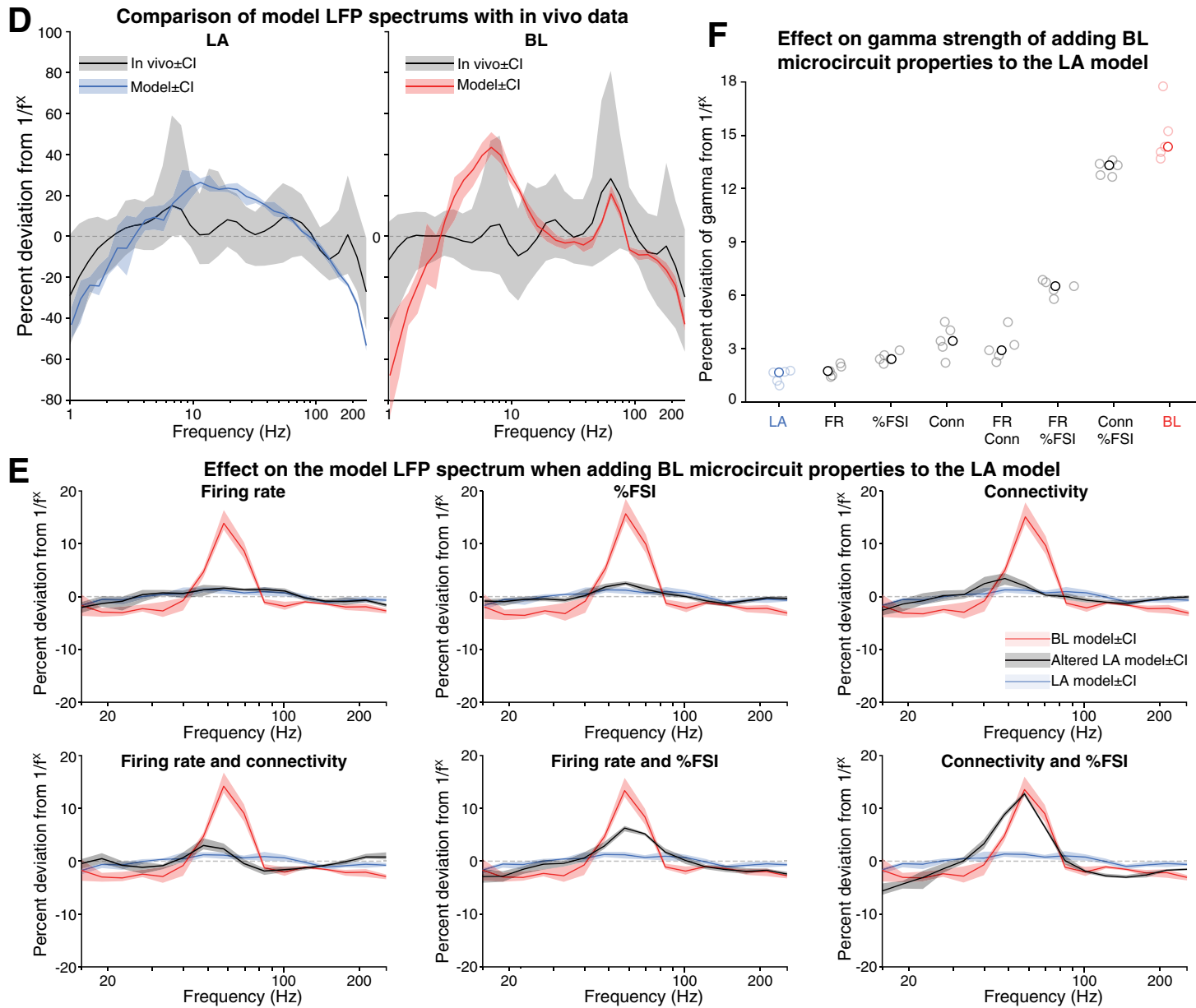

Figure 4. Effect of microcircuit properties on gamma generation in LA and BL. A, Proportion of neurons that were identified as FSIs based on extracellular spiking characteristics. $\boldsymbol{B}$, Distribution of mean firing rates as a function of region and cell type. C1, Overall probabilities for each type of putative monosynaptic connection as determined by cross-correlation analyses. C2-C4, The probability of each connection decreased with distance between the presynaptic and postsynaptic units. Fits are exponential curves, shaded region denotes $95 \%$ confidence intervals for the fits, and crosses are the measured probabilities. $\boldsymbol{D}$, Biophysically detailed models of the LA and BL were constructed based on the microcircuit properties for each nucleus, and then the LFP was simulated. The deviation in gamma power from $1 / f^{x}$ in both models fell within the $95 \%$ confidence intervals for what we observed in vivo (sold lines are medians; shaded lines are 95\% confidence intervals). $\boldsymbol{E}$, The parameters of the LA model (blue) systematically changed to bring them closer to the BL model (red) and create six different alternate models (black). The red and blue spectrums are the same across all graphs; only the black, alternate model, spectrums vary. The title of each graph indicates the BL parameter that was implemented in the LA model. "Connectivity" refers to the connection probability between PN $\rightarrow$ FSI and FSI $\rightarrow$ PN, "Firing rate" is the mean firing rate of PNs, and "\%FSI" is the proportion of FSIs. $\boldsymbol{F}$, The percentage deviation of mid-gamma from the $1 / f^{x}$ was measured for each model. Each model was simulated five times, shown by the faded circles. The darker circles correspond to the median.

behavioral correlates. LFP recordings were obtained from a subset of our subjects $(n=3)$ during early training, which allowed us to examine how acquiring appetitive and aversive contingencies influenced gamma in LA and BL. We defined two stages of training, early and late (up to vs after the ninth training session, respectively). We excluded the first four training sessions because subjects often behaved in a manner that produced artifact-ridden data. Behaviorally, the early and late stages corresponded to 
mean performances of $56 \%$ and $76 \%$ correct trials for all CS types combined, respectively. We considered four to five early and four to five late training sessions per rat $(n=3)$, during which the same $61 \mathrm{BL}$ and 30 LA recording sites were monitored $(\geq 19 \mathrm{BL}$ and $7 \mathrm{LA}$ sites per rat).

We first compared LFP power spectrums during the ITIs, at early (Fig. $5 \mathrm{~A}$, black traces) versus late stages of learning (Fig. 5, colored traces). To remove individual differences in overall LFP power and focus on narrow-band peaks in the power spectrum, we expressed the magnitude of each frequency as the deviation from the $1 / f^{x}$ fit of the power spectrum. Because adjacent frequencies tended to exhibit similar changes in power, we used mass univariate statistics to assess the significance of spectral changes (see Materials and Methods). The range of frequencies whose power changed significantly with learning is indicated at the top of the graph (darkness proportional to $F$ statistics, all significant at $p<0.05$ ). While changes were observed across numerous frequency bands, the following results focus on the mid-gamma band.

LA (Fig. 5A, left) and BL (Fig. 5A, right) showed a similar pattern of learning-induced changes in spectral power. From early to late learning stages, LFP power during the ITIs reduced in the mid-gamma band, along with adjacent bands. Notably, LA exhibited a gamma peak at early stages of training, in contrast to subjects shown earlier (Fig. 1B), who were at behavioral asymptote. The learning-related decrease in gamma during the ITIs suggests that baseline gamma levels are elevated in LA and BL when subjects are uncertain about the task structure.

\section{Learning-related changes in oscillatory} activity during conditioned stimuli

Having assessed how learning alters gamma activity during the ITI, we next considered changes induced by the CS-S (Fig. 5B) and CS-R (Fig. 5C) relative to the last $5 \mathrm{~s}$ of the ITIs. Again, similar results were obtained in LA and BL. Early in training, a distinct peak in the mid-gamma range was evident, centered at $53 \mathrm{~Hz}$. Further training reduced this bump significantly (Fig. 5B; LA, $p<0.001$; $\mathrm{BL}, p<0.001$ ). A similar pattern was obtained with the CS-R (Fig. $5 C$ ), except for the fact that the mid-gamma peak was wider and the learning-related decrease in gamma power was so pronounced (LA, $p<0.0 .001$; $\mathrm{BL}, p<0.001)$ that it fell below ITI levels. Altogether, these results indicate that LA and BL exhibit elevated gamma levels early in training and that these are reduced with learning.

\section{Oscillatory correlates of conditioned stimuli and behaviors in $B L$ and $L A$}

On the surface, the above seems to suggest that once rats have become proficient at this task, mid-gamma is largely absent during aversive and appetitive CSs. However, examination of moment-to-moment variations in the spectral composition of LFPs in fact revealed that mid-gamma is associated with specific behavioral transitions. Here, the reader is reminded that in contrast with standard conditioning paradigms, the RRI task allows for multiple CRs. During aversive trials, depending on the position of rats with respect to the shock sector, the CS-S called for PA (64\%) or AA (36\%) responses. Behavioral variability was especially pronounced on CS-S trials that required an AA response. In $8 \%$ of these trials, rats failed to move away from the shock sector, instead freezing until shock onset. In $17 \%$ of cases, after a bout of freezing, they generated the appropriate AA response. Finally, in $75 \%$ of cases, rats left the shock sector without first engaging in freezing behavior.

To study the oscillatory correlates of these behavioral variations, for each trial type we separately averaged LFP spectrograms, removed the $1 / f^{x}$ background, and $z$-scored the data to the last $5 \mathrm{~s}$ of the ITI (Fig. 6, left column). Because the latencies and durations of the various CRs varied from trial to trial, averaging across trials required that the data be binned as a function of time relative to behavioral events. As detailed in the Materials and Methods section, this was achieved by distributing a fixed number of time bins across the various behavioral phases. To assess the statistical significance of power changes, we performed 

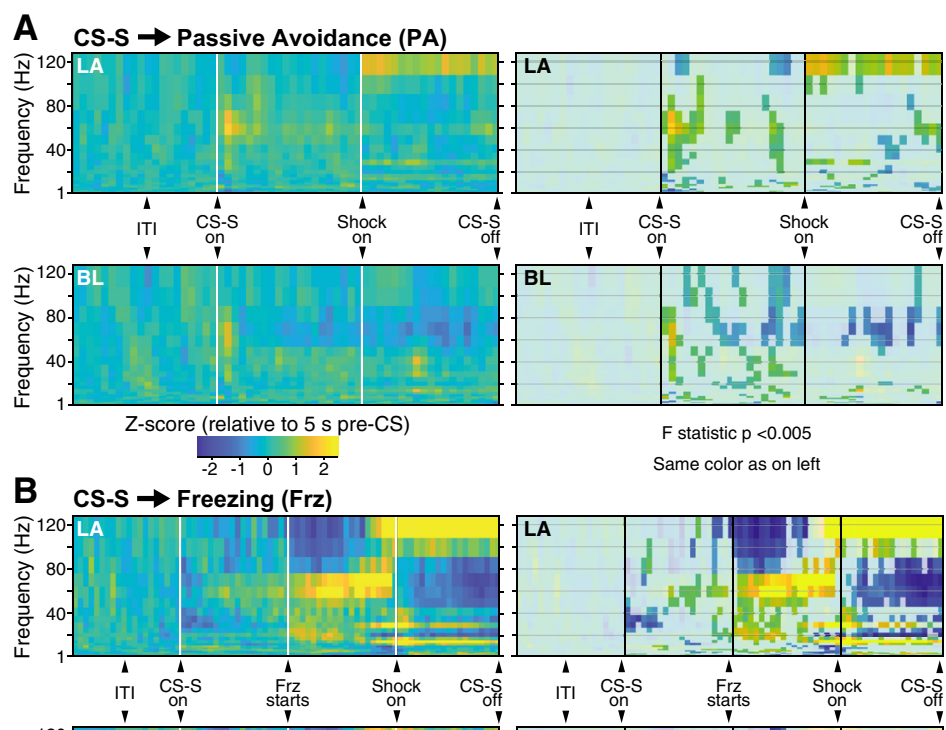

Same color as on left
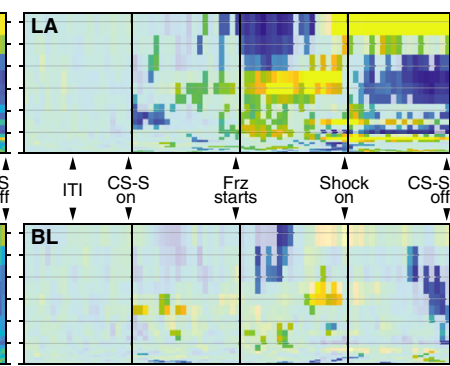

C CS-S $\rightarrow$ Freezing (Frz) $\rightarrow$ Active Avoidance (AA)
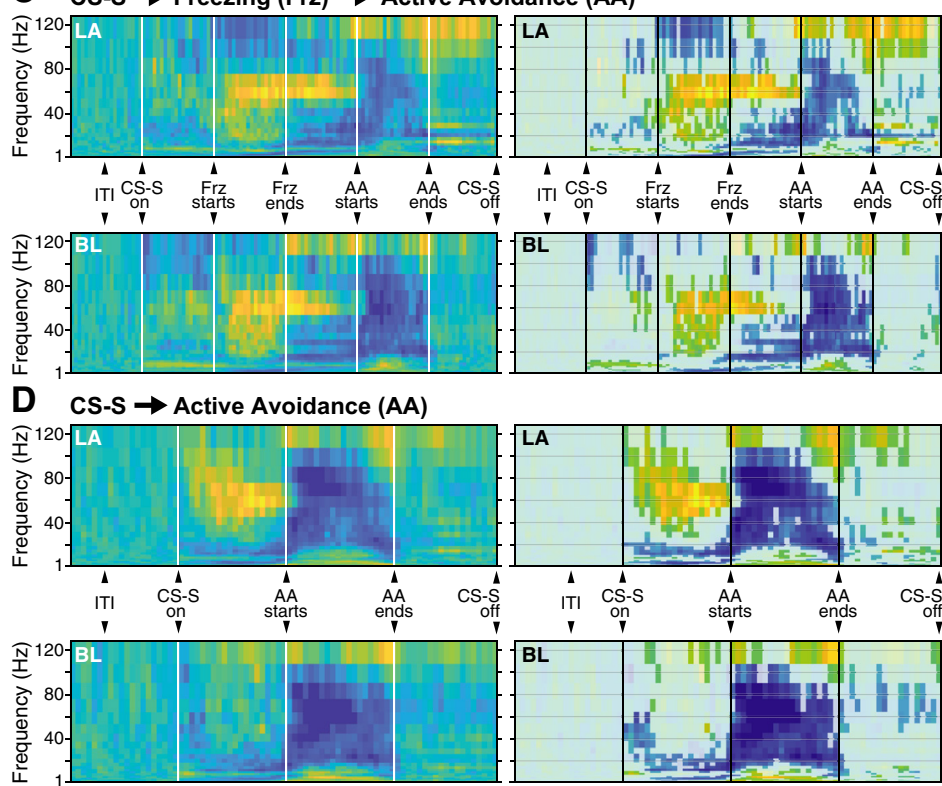

Figure 6. Behavioral correlates of mid-gamma activity during CS-S trials. A-D, Left, Average spectrograms across recording sites $z$-scored to the last $5 \mathrm{~s}$ of ITI and plotted as a function of relative time. Data obtained in $L A$ and $B L$ is shown at the top and bottom, respectively. $\boldsymbol{A}-\boldsymbol{D}$, Right, Same spectrograms as on left, but statistically significant bins have the same color as on the left. Light shaded bins were not statistically significant. $\boldsymbol{A}$, Trials where the CS-S called for a PA response because rats were outside the shock sector. $\boldsymbol{B}$, Trials where the CS-S elicited behavioral freezing up to unconditioned stimulus delivery. $\boldsymbol{C}$, Trials where the CS-S first elicited freezing and, after a delay, AA. D, Trials where the CS-S elicited AA with no preceding freezing.

$F$ tests on each time-frequency bin relative to the corresponding ITI baseline and calculated the $p$ values of those using mass univariate statistics. In the right column of Figure 6, significant bins have the same color as on the left, whereas nonsignificant ones are faded $(p>0.05)$.

On passive avoidance trials, when no action was required to avoid the shock, the CS-S onset elicited modest and short-lived increases in mid-gamma power $(n=77 \mathrm{BL}$ sites; $n=105$ LA sites; Fig. 6A). In contrast, during CS-S trials where rats exhibited behavioral freezing up to shock delivery $(n=28 \mathrm{BL}$ sites; $n=78$ LA sites; Fig. $6 B$ ), mid-gamma power increased modestly before freezing in LA and $\mathrm{BL}$, ramped up further during freezing, peaking just before shock delivery, after which it decreased below ITI levels as rats escaped from the shock sector.

Although these results give the impression that mid-gamma power is associated with freezing, the case where rats stopped freezing early enough during the CS-S to actively avoid the shock indicates otherwise $(n=77 \mathrm{BL}$ sites; $n=99$ LA sites; Fig. 6C). On these trials, while mid-gamma increased during freezing, it persisted shortly after the rats began moving again, and then dropped below ITI levels during active avoidance. In addition, increased mid-gamma during freezing started earlier in LA than BL (mass univariate test with region as a factor, $p<0.005$ ).

This suggests that LA and BL could differentially express mid-gamma during aversive behaviors. Indeed, gamma increases were sometimes restricted to just one of the two nuclei. On CS-S trials where rats did not engage in freezing before avoiding the shock sector ( $n=77$ BL sites; $n=105$ LA sites; Fig. $6 D)$, mid-gamma ramped up only in LA before avoidance $(p<0.005)$, then abruptly dropped below ITI levels during avoidance, and returned to baseline values once rats reached safety. Altogether, these results indicate that gamma in LA and BL is associated with threat assessment and response selection, especially given that freezing is a behavioral manifestation of both processes (Lojowska et al., 2015; Gladwin et al., 2016).

However, it may be that gamma activity in LA and $\mathrm{BL}$ is not specific for threatening situations, but instead reflects environmental assessment and action selection more generally. To test this, we examined appetitive trials (Fig. 7). On CS-R trials where rats were close to the CS-R, they simply placed their front paws on the water port and waited for reward delivery (32.1\% of trials), a behavior termed "reward anticipation." On other trials (63.1\%), when rats were far from the CS-R, they first ran to the water port (a behavior termed "reward approach") and then exhibited reward anticipation. On a minority of trials, rats failed to approach the water port before the end of the CS-R ( $4.8 \%$ of trials).

On appetitive trials where rats were adjacent to the water port ( $n=69 \mathrm{BL}$ sites; $n=104 \mathrm{LA}$ sites; Fig. $7 A$ ), mid-gamma rose modestly after CS-R onset, decreased after the start of reward anticipation, and dropped well below ITI levels upon reward delivery. On trials where rats were far from the water port $(n=77$ BL sites; $n=105$ LA sites; Fig. $7 B)$, midgamma power rose transiently after CS-R onset, then again immediately before the start of reward approach, and dropped drastically during reward approach, anticipation, and delivery. On trials where subjects did not interact with the port, gamma power did not change $(n=38 \mathrm{BL}$ sites; $n=94$ LA sites; Fig. $7 C$ ). Taken with the results from aversive trials, these findings suggest that BLA mid-gamma supports a process akin to deliberation or response selection, regardless of the valence of the situation. 


\section{Discussion}

This study examined the generation and behavioral correlates of gamma oscillations in the BLA. Both the LA and BL exhibited gamma oscillations during stimulus presentation and specific behavioral periods. Gamma in LA and BL is associated with behavioral epochs reflecting environmental assessment and response selection. However, only BL showed spontaneous gamma oscillations during quiet waking or intertrial periods. This arises from an interplay between its distinctive microcircuit characteristics.

\section{BL microcircuitry is conducive to gamma generation}

During rest and RRI-task ITIs, spontaneous gamma oscillations were readily detected in the BL nucleus, but not in LA. While we cannot exclude that volume conduction from adjacent cortices contributed to this gamma (Carmichael et al., 2017), much of it surely depended on local neuronal activity since it entrained local spiking and it was present in the power spectrum derived from locally generated currents. Further indicating that volume conduction was not a major contributor to the gamma we observed in BLA, spontaneous gamma bursts expressed in BL were not readily apparent in LA (Fig. 1A), and task-evoked gamma could occur in LA but not BL (Fig. 6B).

What factors account for the propensity of BL to generate gamma? While LA and $\mathrm{BL}$ exhibit the same microcircuit motifs, such as dense reciprocal connections between PNs and FSIs (Woodruff and Sah, 2007), they differ in critical ways like the higher incidence of PV cells in BL than LA (Smith et al., 1998; McDonald and Mascagni, 2001; Butler et al., 2018), and PV cells in LA mediating more feedforward inhibition than their BL counterparts (Lucas et al., 2016). We identified the following three differences between BL and LA: a higher incidence of FSIs in BL;

denser interconnections between PNs and FSIs in BL; and a trend for PNs to fire at higher rates in BL than LA. Incorporating these differing properties into biophysically detailed models of LA and BL reproduced their differing gamma levels. Critically, no single microcircuit feature was responsible for this difference. In general, gamma was unaffected by $\mathrm{PN}$ firing rates, but increased with denser connectivity between PNs and FSIs and the proportion of FSIs.

\section{Origin of LA task-related gamma}

LA only exhibited gamma during task events, particularly at early stages of training. Since our biophysical model revealed that the microcircuitry of LA is not conducive to gamma generation, how might it occur during task events? One explanation is that
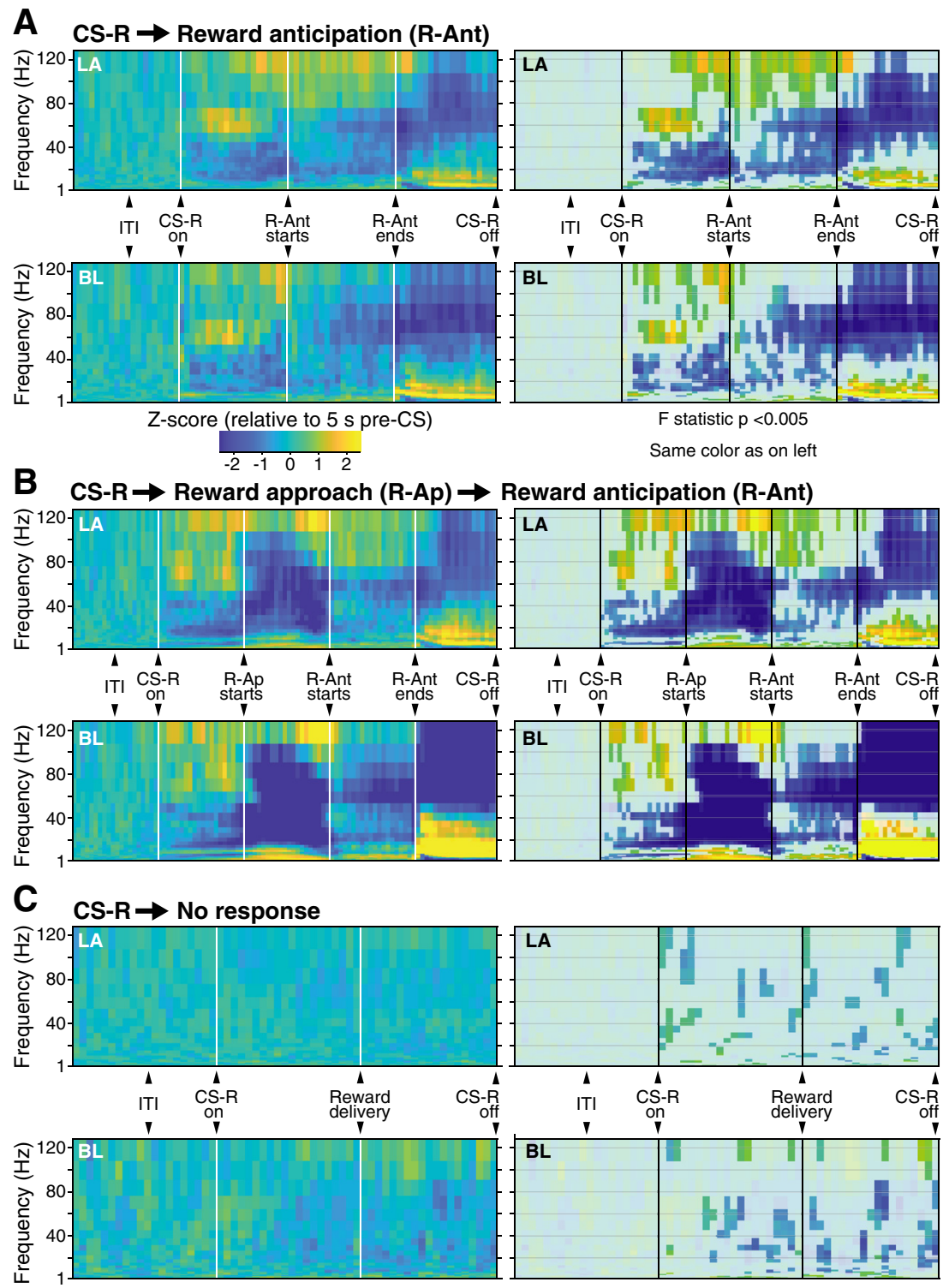

Figure 7. Behavioral correlates of mid-gamma activity during CS-R trials. A-C, Left, Average spectrograms across recording sites $z$-scored to the last $5 \mathrm{~s}$ of the ITI and plotted as a function of relative time. Data obtained in LA and BL is shown at the top and bottom, respectively. $\boldsymbol{A}-\boldsymbol{C}$, Right, Same spectrograms as on left but statistically significant bins have the same cor as on the left. Light shaded bins were not statistically significant. $A$, Trials where the CS-R only elicited reward anticipation (R-Ant) because rats were adjacent to the water port. $\boldsymbol{B}$, Trials where the CS-R first elicited reward approach and then R-Ant. C, Trials where the CS-R elicited no response.

sufficient extrinsic activation drives PNs enough to engage the PING mechanism. Or extrinsic inputs that target FSIs (Woodson et al., 2000) drive them to generate gamma autonomously (Tiesinga and Sejnowski, 2009). Or structures upstream of LA may impose gamma oscillations (Headley and Weinberger, 2013). Last, neuromodulation of LA may induce gamma by altering the excitability of particular subsets of neurons or the properties of synaptic transmission (Fisahn et al., 2002; Sinfield and Collins, 2006; Gire and Schoppa, 2008; Andersson et al., 2012; McCall et al., 2017).

\section{BL gamma modulates excitatory synaptic transmission}

The principal function of gamma is thought to be the regulation of communication between ensembles of neurons (Fries, 2015). Each gamma cycle is composed of excitation followed by 
inhibition so that inputs arriving during the excitatory phase exert a greater influence on the postsynaptic neuron than those coinciding with the inhibitory phase. Gamma phase affects the coordination of multiunit activity in auditory cortex (Headley and Weinberger, 2013), single unit in amygdala (Amir et al., 2018), and stimulus responding in visual cortex (Ni et al., 2016). In BLA, multiunit cross-correlations are enhanced with gamma (Stujenske et al., 2014). However, it is unclear whether the effect was driven by a change in the integration of individual synaptic events or a shift in the set of neurons firing. Intervention studies established that rhythmic inhibition in the gamma frequency range creates optimal phases for receptiveness to stimuli (Cardin et al., 2009), with corresponding behavioral effects (Siegle et al., 2014), but these were artificially generated rhythms. Here, we directly tested whether gamma regulates neuronal communication by studying how the impact of monosynaptic connections changes as a function of gamma phase. We found that the excitatory phase of gamma enhances the responsiveness of postsynaptic neurons to excitatory inputs, providing experimental confirmation that gamma regulates communication between neurons (Fries, 2015).

Crucially, this phenomenon was found in BL, but not LA, which lacked gamma oscillations during the same periods. This highlights the importance of gamma itself in modulating neuronal responsiveness. Alternatively, a mundane explanation is that since the LFP reflects local excitability (Haider et al., 2006; Okun et al., 2010), it would necessarily correlate with neuronal responsiveness. Thus, it is conceivable that gamma-phase modulation of responsiveness may have arisen because the LFP phase in the gamma frequency range reflects local excitability, regardless of the presence of gamma. Arguing against that, we should have observed a similar modulation in LA and BL, which we did not.

\section{What is the function of BLA gamma?}

Given that LA and BL are reciprocally connected with numerous cortical areas (Krettek and Price, 1977; McDonald, 1998), the occurrence of gamma may support their interactions during different behavioral states.

Starting with online states, during acquisition of the RRI task, gamma oscillations were prominent in LA and BL, but further training diminished them. Despite this reduction, gamma oscillations still occurred, concentrating around particular behavioral events, namely freezing, and the initiation of active behaviors. These findings suggest that gamma oscillations during acquisition reflect the uncertainty of the rats about task contingencies and increased attention to the environment. Indeed, freezing is considered a behavioral manifestation of environmental assessment (Lojowska et al., 2015; Gladwin et al., 2016), a process that likely entails coordination of cortical and amygdala activity. LA has dense connections with the perirhinal cortex (Pitkänen et al., 2000), a multimodal integration site capable of persistent spiking in the absence of stimuli, so coordination of perirhinal and LA activity by gamma may support processing of complex stimuli or contingencies (Kent and Brown, 2012). Crucially, the perirhinal cortex and LA can exhibit coherent gamma-band activities (Collins et al., 2001; Bauer et al., 2007).

Gamma also increased immediately before the initiation of active behaviors. This may be the source of mid-gamma observed in striatum during movement initiation (Massimore et al., 2005). Indeed, LA and BL project to the striatum (Kita and Kitai, 1990; McDonald, 1991), and during a cued reward task they show coherent gamma with striatum, which was disrupted by BLA inactivation (Popescu et al., 2009). More closely related to the
RRI task, BLA projections to the striatum have also been implicated in the generation of active avoidance (Ramirez et al., 2015; Diehl et al., 2020).

After the initiation of active CRs, mid-gamma power decreased drastically. Since movement artifacts would be expected to have the opposite effect and we confirmed that the signal did not saturate our recording system, the drop in midgamma power is likely true. At present, its origin is unclear. One possibility is that it reflects a reduction in extrinsic synaptic drive. Consistent with this possibility, we previously observed that most BL neurons decrease their firing rate during movements, whether when foraging for food or exploring an open field (Amir et al., 2015).

Finally, with respect to the function of BL gamma during offline states (e.g., the ITI period in this study), one possibility is that it supports the well established role of BL in the emotional modulation of memory consolidation (McGaugh, 2000). Of note, $\mathrm{BL}$ has prominent reciprocal connections with the CA1 field of the ventral hippocampus (Pitkänen et al., 2000), an area crucial to memory formation. Tying this to gamma, artificial enhancement or reduction of $\mathrm{BL}$ gamma during postlearning rest has corresponding effects on memory strength (Kanta et al., 2019). Another possibility is that gamma supports the interactions of $\mathrm{BL}$ with the hippocampus, a pathway that is important for planning in anxiogenic situations (Felix-Ortiz et al., 2013; Wu et al., 2017), when threats are uncertain. Another structure important in such situations is the prelimbic cortex. It has reciprocal connections with the BL (Gabbott et al., 2005; Hoover and Vertes, 2007), with which it exhibits bursts of coherent gamma (Stujenske et al., 2014). This prefrontal subregion is crucial for freezing behavior (Sierra-Mercado et al., 2011), and it exhibits mixed selectivity (Kyriazi et al., 2020), which supports the higher-order task representations thought to underlie strategic behaviors (Fusi et al., 2016). Consequently, BL gamma may reflect its interactions with these structures in support of ruminative processing and evaluation of complex situations.

\section{Conclusion}

Previous studies lumped LA and BL together when investigating gamma. Our results indicate that this may mask important differences in the function of gamma between these structures and in the organization of their microcircuitry. Since the BLA likely interacts with shifting sets of systems during different phases of emotionally salient situations (Mobbs et al., 2020), the gamma oscillations we observed may support these interactions.

\section{References}

Aarts E, Verhage M, Veenvliet JV, Dolan CV, van der Sluis S (2014) A solution to dependency: using multilevel analysis to accommodate nested data. Nat Neurosci 17:491-496.

Alturki A, Feng F, Nair A, Guntu V, Nair SS (2016) Distinct current modules shape cellular dynamics in model neurons. Neuroscience 334:309-331.

Amir A, Lee SC, Headley DB, Herzallah MM, Pare D (2015) Amygdala signaling during foraging in a hazardous environment. J Neurosci 35:12994-13005.

Amir A, Headley DB, Lee SC, Haufler D, Paré D (2018) Vigilance-associated gamma oscillations coordinate the ensemble activity of basolateral amygdala neurons. Neuron 97:656-669.

Andersson RH, Johnston A, Herman PA, Winzer-Serhan UH, Karavanova I, Vullhorst D, Fisahn A, Buonanno A (2012) Neuregulin and dopamine modulation of hippocampal gamma oscillations is dependent on dopamine D4 receptors. Proc Natl Acad Sci U S A 109:13118-13123.

Atallah BV, Scanziani M (2009) Instantaneous modulation of gamma oscillation frequency by balancing excitation with inhibition. Neuron 62:566577. 
Barthó P, Hirase H, Monconduit L, Zugaro M, Harris KD, Buzsáki G (2004) Characterization of neocortical principal cells and interneurons by network interactions and extracellular features. J Neurophysiol 92:600-608.

Bauer EP, Paz R, Paré D (2007) Gamma oscillations coordinate amygdalorhinal interactions during learning. J Neurosci 27:9369-9379.

Berke JD (2008) Uncoordinated firing rate changes of striatal fast-spiking interneurons during behavioral task performance. J Neurosci 28:1007510080.

Butler RK, Oliver EM, Fadel JR, Wilson MA (2018) Hemispheric differences in the number of parvalbumin-positive neurons in subdivisions of the rat basolateral amygdala complex. Brain Res 1678:214-219.

Buzsáki G, Wang X-J (2012) Mechanisms of gamma oscillations. Annu Rev Neurosci 35:203-225.

Cardin JA, Carlén M, Meletis K, Knoblich U, Zhang F, Deisseroth K, Tsai LH, Moore CI (2009) Driving fast-spiking cells induces gamma rhythm and controls sensory responses. Nature 459:663-667.

Carmichael JE, Gmaz JM, van der Meer MAA (2017) Gamma oscillations in the rat ventral striatum originate in the piriform cortex. J Neurosci 37:7962-7974

Carnevale NT, Hines ML (2005) The neuron book. New York: Cambridge UP.

Chareyron LJ, Banta Lavenex P, Amaral DG, Lavenex P (2011) Stereological analysis of the rat and monkey amygdala. J Comp Neurol 519:3218-3239.

Collins DR, Pelletier JG, Paré D (2001) Slow and Fast (Gamma) Neuronal oscillations in perirhinal cortex and lateral amygdala. J Neurophysiol 85:1661-1672.

Courtin J, Karalis N, Gonzalez-Campo C, Wurtz H, Herry C (2014) Persistence of amygdala gamma oscillations during extinction learning predicts spontaneous fear recovery. Neurobiol Learn Mem 113:82-89.

Destexhe A, Rudolph M, Fellous JM, Sejnowski TJ (2001) Fluctuating synaptic conductances recreate in vivo-like activity in neocortical neurons. Neuroscience 107:13-24.

Diehl MM, Iravedra-Garcia JM, Morán-Sierra J, Rojas-Bowe G, GonzalezDiaz FN, Valentín-Valentín VP, Quirk GJ (2020) Divergent projections of the prelimbic cortex bidirectionally regulate active avoidance. Elife 9: e59281.

English DF, McKenzie S, Evans T, Kim K, Yoon E, Buzsáki G (2017) Pyramidal cell-interneuron circuit architecture and dynamics in hippocampal networks. Neuron 96:505-520.

Faber ES, Callister RJ, Sah P (2001) Morphological and electrophysiological properties of principal neurons in the rat lateral amygdala in vitro. J Neurophysiol 85:714-723.

Felix-Ortiz AC, Beyeler A, Seo C, Leppla CA, Wildes CP, Tye KM (2013) BLA to vHPC inputs modulate anxiety-related behaviors. Neuron 79:658-664

Feng F, Headley DB, Amir A, Kanta V, Chen Z, Paré D, Nair SS (2019) Gamma oscillations in the basolateral amygdala: biophysical mechanisms and computational consequences. eNeuro 6:ENEURO.0388-18.2018.

Fisahn A, Yamada M, Duttaroy A, Gan J-W, Deng C-X, McBain CJ, Wess J (2002) Muscarinic induction of hippocampal gamma oscillations requires coupling of the M1 receptor to two mixed cation currents. Neuron 33:615-624.

Fisher NI (1995) Statistical analysis of circular data. New York: Cambridge UP.

Fries P (2015) Rhythms for cognition: communication through coherence. Neuron 88:220-235.

Fusi S, Miller EK, Rigotti M (2016) Why neurons mix: high dimensionality for higher cognition. Curr Opin Neurobiol 37:66-74.

Gire DH, Schoppa NE (2008) Long-term enhancement of synchronized oscillations by adrenergic receptor activation in the olfactory bulb. J Neurophysiol 99:2021-2025.

Haider B, Duque A, Hasenstaub AR, McCormick DA (2006) Neocortical network activity in vivo is generated through a dynamic balance of excitation and inhibition. J Neurosci 26:4535-4545.

Harrison D, Kanji GK (1988) The development of analysis of variance for circular data. J Appl Stat 15:197-223.

Hasenstaub A, Shu Y, Haider B, Kraushaar U, Duque A, McCormick DA (2005) Inhibitory postsynaptic potentials carry synchronized frequency information in active cortical networks. Neuron 47:423-435.

Hazan L, Zugaro M, Buzsáki G (2006) Klusters, NeuroScope, NDManager: a free software suite for neurophysiological data processing and visualization. J Neurosci Methods 155:207-216.
Headley DB, Weinberger NM (2013) Fear conditioning enhances $\gamma$ oscillations and their entrainment of neurons representing the conditioned stimulus. J Neurosci 33:5705-5717.

Hill DN, Mehta SB, Kleinfeld D (2011) Quality metrics to accompany spike sorting of extracellular signals. J Neurosci 31:8699-8705.

Hoover WB, Vertes RP (2007) Anatomical analysis of afferent projections to the medial prefrontal cortex in the rat. Brain Struct Funct 212:149-179.

Huff ML, Miller RL, Deisseroth K, Moorman DE, LaLumiere RT (2013) Posttraining optogenetic manipulations of basolateral amygdala activity modulate consolidation of inhibitory avoidance memory in rats. Proc Natl Acad Sci U S A 110:3597-3602.

Gabbott PL, Warner TA, Jays PR, Salway P, Busby SJ (2005) Prefrontal cortex in the rat: projections to subcortical autonomic, motor, and limbic centers. J Comp Neurol 492:145-177.

Gladwin TE, Hashemi MM, van Ast V, Roelofs K (2016) Ready and waiting: freezing as active preparation under threat. Neurosci Lett 619:182-188.

Joshua M, Elias S, Levine O, Bergman H (2007) Quantifying the isolation quality of extracellularly recorded action potentials. J Neurosci Methods 163:267-282.

Kanta V, Pare D, Headley DB (2019) Closed-loop control of gamma oscillations in the amygdala demonstrates their role in spatial memory consolidation. Nat Commun 10:3970.

Kent BA, Brown TH (2012) Dual functions of perirhinal cortex in fear conditioning. Hippocampus 22:2068-2079.

Kita H, Kitai ST (1990) Amygdaloid projections to the frontal cortex and the striatum in the rat. J Comp Neurol 298:40-49.

Krettek JE, Price JL (1977) Projections from the amygdaloid complex to the cerebral cortex and thalamus in the rat and cat. J Comp Neurol 172:687722.

Kyriazi P, Headley DB, Pare D (2018) Multi-dimensional coding by basolateral amygdala neurons. Neuron 99:1315-1328.

Kyriazi P, Headley DB, Paré D (2020) Different multidimensional representations across the amygdalo-prefrontal network during an approach-avoidance task. Neuron 107:717-730.

LeDoux JE (2000) Emotion circuits in the brain. Annu Rev Neurosci 23:155184.

Łẹski S, Pettersen KH, Tunstall B, Einevoll GT, Gigg J, Wójcik DK (2011) Inverse current source density method in two dimensions: inferring neural activation from multielectrode recordings. Neuroinformatics 9:401425 .

Linden H, Tetzlaff T, Potjans TC, Pettersen KH, Grun S, Diesmann M, Einevoll GT (2011) Modeling the spatial reach of the LFP. Neuron 72:859-872.

Lojowska M, Gladwin TE, Hermans EJ, Roelofs K (2015) Freezing promotes perception of coarse visual features. J Exp Psychol Gen 144:1080-1088.

Lucas EK, Jegarl AM, Morishita H, Clem RL (2016) Multimodal and site-specific plasticity of amygdala parvalbumin interneurons after fear learning. Neuron 91:629-643.

Luo Q, Holroyd T, Jones M, Hendler T, Blair J (2007) Neural dynamics for facial threat processing as revealed by gamma band synchronization using MEG. Neuroimage 34:839-847.

Luo Q, Mitchell D, Cheng X, Mondillo K, Mccaffrey D, Holroyd T, Carver F, Coppola R, Blair J (2009) Visual awareness, emotion, and gamma band synchronization. Cereb Cortex 19:1896-1904.

Massimore B, Schmitzer-Torbert NC, Kakalios J, Redish AD (2005) Transient striatal gamma local field potentials signal movement initiation in rats. Neuroreport 16:2021-2024.

McCall JG, Siuda ER, Bhatti DL, Lawson LA, McElligott ZA, Stuber GD, Bruchas MR (2017) Locus coeruleus to basolateral amygdala noradrenergic projections promote anxiety-like behavior. eLife 6:e18247.

McDonald AJ (1991) Organization of amygdaloid projections to the prefrontal cortex and associated striatum in the rat. Neuroscience 44:1-14.

McDonald AJ (1998) Cortical pathways to the mammalian amygdala. Prog Neurobiol 55:257-332.

McDonald AJ, Mascagni F (2001) Colocalization of calcium-binding proteins and GABA in neurons of the rat basolateral amygdala. Neuroscience 105:681-693.

McGaugh JL (2000) Memory-a century of consolidation. Science 287:248251.

Mobbs D, Headley DB, Ding W, Dayan P (2020) Space, time, and fear: survival computations along defensive circuits. Trends Cogn Sci 24:228-241. 
Ni J, Wunderle T, Lewis CM, Desimone R, Diester I, Fries P (2016) Gammarhythmic gain modulation. Neuron 92:240-251.

Okun M, Naim A, Lampl I (2010) The subthreshold relation between cortical local field potential and neuronal firing unveiled by intracellular recordings in awake rats. J Neurosci 30:4440-4448.

Oswald A-MM, Doiron B, Rinzel J, Reyes AD (2009) Spatial profile and differential recruitment of $\mathrm{GABA}_{\mathrm{B}}$ modulate oscillatory activity in auditory cortex. J Neurosci 29:10321-10334.

Oya H, Kawasaki H, Howard MA 3rd, Adolphs R (2002) Electrophysiological responses in the human amygdala discriminate emotion categories of complex visual stimuli. J Neurosci 22:9502-9512.

Pagano RR, Gault FP (1964) Amygdala activity: a central measure of arousal. Electroencephalogr Clin Neurophysiol 17:255-260.

Parasuram H, Nair B, D'Angelo E, Hines M, Naldi G, Diwakar S (2016) Computational modeling of single neuron extracellular electric potentials and network local field potentials using LFPsim. Front Comput Neurosci 28:65.

Paré D, Gaudreau H (1996) Projection cells and interneurons of the lateral and basolateral amygdala: distinct firing patterns and differential relation to theta and delta rhythms in conscious cats. J Neurosci 16:3334-3350.

Pêgo JM, Morgado P, Pinto LG, Cerqueira JJ, Almeida OF, Sousa N (2008) Dissociation of the morphological correlates of stress-induced anxiety and fear. Eur J Neurosci 27:1503-1516.

Pitkänen A, Pikkarainen M, Nurminen N, Ylinen A (2000) Reciprocal connections between the amygdala and the hippocampal formation, perirhinal cortex, and postrhinal cortex in rat. A review. Ann N Y Acad Sci 911:369-391.

Popescu AT, Popa D, Paré D (2009) Coherent gamma oscillations couple the amygdala and striatum during learning. Nat Neurosci 12:801-807.

Ramirez F, Moscarello JM, LeDoux JE, Sears RM (2015) Active avoidance requires a serial basal amygdala to nucleus accumbens shell circuit. J Neurosci 35:3470-3477.

Rosenberg JR, Amjad AM, Breeze P, Brillinger DR, Halliday DM (1989) The Fourier approach to the identification of functional coupling between neuronal spike trains. Prog Biophys Mol Biol 53:1-31.

Rubinow MJ, Juraska JM (2009) Neuron and glia numbers in the basolateral nucleus of the amygdala from preweaning through old age in male and female rats: a stereological study. J Comp Neurol 512:717-725.

Sah P, Faber ES, Lopez De Armentia M, Power J (2003) The amygdaloid complex: anatomy and physiology. Physiol Rev 83:803-834.

Salkoff DB, Zagha E, Yüzgeç Ö, McCormick DA (2015) Synaptic mechanisms of tight spike synchrony at gamma frequency in cerebral cortex. J Neurosci 35:10236-10251.

Salm AK, Pavelko M, Krouse EM, Webster W, Kraszpulski M, Birkle DL (2004) Lateral amygdaloid nucleus expansion in adult rats is associated with exposure to prenatal stress. Brain Res Dev Brain Res 148:159-167.
Scheffer-Teixeira R, Belchior H, Leão RN, Ribeiro S, Tort ABL (2013) On high-frequency field oscillations $(>100 \mathrm{~Hz})$ and the spectral leakage of spiking activity. J Neurosci 33:1535-1539.

Siegle JH, Pritchett DL, Moore CI (2014) Gamma-range synchronization of fast-spiking interneurons can enhance detection of tactile stimuli. Nat Neurosci 17:1371-1379.

Sierra-Mercado D, Padilla-Coreano N, Quirk GJ (2011) Dissociable roles of prelimbic and infralimbic cortices, ventral hippocampus, and basolateral amygdala in the expression and extinction of conditioned fear. Neuropsychopharmacology 36:529-538.

Sinfield JL, Collins DR (2006) Induction of synchronous oscillatory activity in the rat lateral amygdala in vitro is dependent on gap junction activity. Eur J Neurosci 24:3091-3095.

Sivagnanam S, Majumdar A, Yoshimoto K, Astakhov V, Bandrowski A, Martone ME, Carnevale NT (2013) Introducing the neuroscience gateway. CEUR Workshop Proc 993:10.

Smith Y, Paré J-F, Paré D (1998) Cat intraamygdaloid inhibitory network: ultrastructural organization of parvalbumin-immunoreactive elements. J Comp Neurol 391:164-179.

Stark E, Abeles M (2009) Unbiased estimation of precise temporal correlations between spike trains. J Neurosci Methods 179:90-100.

Stujenske JM, Likhtik EK, Topiwala MA, Gordon JA (2014) Fear and safety engage competing patterns of theta-gamma coupling in the basolateral amygdala. Neuron 83:919-933.

Tiesinga P, Sejnowski TJ (2009) Cortical enlightenment: are attentional gamma oscillations driven by ING or PING? Neuron 63:727-732.

Torrence C, Compo GP (1998) A practical guide to wavelet analysis. Bull Am Meteor Soc 79:61-78.

Tuunanen J, Pitkänen A (2000) Do seizures cause neuronal damage in rat amygdala kindling? Epilepsy Res 39:171-176.

van der Meer MAA, Kalenscher T, Lansink CS, Pennartz CMA, Berke JD, Redish DA (2010) Integrating early results on ventral striatal gamma oscillations in the rat. Front Neurosci 4:300.

Vinck M, van Wingerden M, Womelsdorf T, Fries P, Pennartz CMA (2010) The pairwise phase consistency: a bias-free measure of rhythmic neuronal synchronization. Neuroimage 51:112-122.

Whittington MA, Traub RD, Kopell N, Ermentrout B, Buhl EH (2000) Inhibition-based rhythms: experimental and mathematical observations on network dynamics. Int J Psychophysiol 38:315-336.

Woodruff AR, Sah P (2007) Networks of parvalbumin-positive interneurons in the basolateral amygdala. J Neurosci 27:553-563.

Woodson W, Farb CR, Ledoux JE (2000) Afferents from the auditory thalamus synapse on inhibitory interneurons in the lateral nucleus of the amygdala. Synapse 38:124-137.

Wu C-T, Haggerty D, Kemere C, Ji D (2017) Hippocampal awake replay in fear memory retrieval. Nat Neurosci 20:571-580.

Zanos TP, Mineault PJ, Pack CC (2011) Removal of spurious correlations between spikes and local field potentials. J Neurophysiol 105:474-486. 